ThE ASTROPHYSiCAL Journal, 329:532-550, 1988 June 15

(C) 1988. The American Astronomical Society. All rights reserved. Printed in U.S.A.

\title{
THE OPTICAL CONTINUA OF EXTRAGALACTIC RADIO JETS ${ }^{1}$
}

\author{
William C. KeEL \\ Sterrewacht Leiden \\ Received 1987 August 11; accepted 1987 December 11
}

\begin{abstract}
Multicolor optical images have been used to measure the broad-band spectral shapes of radio jets known to show optical counterparts, and to search for such counterparts in additional objects. One new detection, the brightest knot in the NGC 6251 jet, is reported. In all cases, except part of the 3C 273 jet, the jet continua are well-represented by synchrotron spectra from truncated power-law electron distributions, with emitted-frame critical frequencies between $3 \times 10^{14}$ and $2 \times 10^{15} \mathrm{~Hz}$. This may be due to fine structure in the jets or to the particle (re)acceleration process. Two hot spots (Pic A and 3C 303) differ from the jets, in showing unbroken power-law spectra from the radio to the $B$ band.

Image restoration has been used to examine the sub-arcsecond structure of the M87 jet and derive spectral shapes for various knots free of blending problems. The structure matches that seen in the radio to the 0 ". 3 level, implying that the jet structure prevents particles from streaming freely over this distance $(\sim 30 \mathrm{pc})$. Significant changes in turnover frequency occur from knot to knot, with an overall trend of higher frequency closer to the nucleus. Knot $\mathrm{A}$ is an exception, with a spectrum like the features near the core.

An Appendix gives results of surface photometry for the program galaxies.

Subject headings: galaxies: individual (M87) - galaxies: jets - galaxies: photometry - quasars radiation mechanisms - radio sources: galaxies
\end{abstract}

\section{INTRODUCTION}

The radio jets of many active galactic nuclei continue to pose outstanding astrophysical problems. Their radio emission is almost certainly dominated by synchrotron radiation, as seen from energy considerations and polarization properties (see the review by Bridle and Perley 1984). The jets, on kiloparsec scales, are characterized by opening angles of a few degrees, clumpy structure, and power-law spectral shapes in the centimeter-wavelength regime, implying a power-law energy distribution for the radiating electrons. Optical, infrared, and ultraviolet observations are in principle of considerable value in determining the properties of the electron distributions at much higher energies (up to $\mathrm{GeV}$ ), but are generally quite difficult due to the low surface brightness of most jets at these wavelengths. The M87 jet is by far the brightest and has been measured by many workers (see $\S$ III). The optical and IR radiation from this jet roughly continues the radio power law, with a falloff toward high frequencies suggesting an upper limit or break in the electron energy spectrum. Similar conclusions are more fragmentary for other objects. The jet of 3C 273 has multiple components, spatially and spectrally distinct, in the optical (Röser and Meisenheimer 1986; Hayes and Sadun 1987). Optical emission from the jets in $3 C$ $31,3 \mathrm{C}$ 66B, and PKS 0521 - 36 has been reported, matching the radio structures and at levels consistent with a synchrotron origin (see $\S$ IV). Individual continuum knots have been identified in the south jet of 3C $277.3=$ Coma A (van Breugel et al. 1985).

Attention has also been given to optical emission from "hot

${ }^{1}$ Based on observations obtained at Kitt Peak National Observatory and Cerro Tololo Inter-American Observatory, National Optical Astronomy Observatories, operated by AURA, Inc., under contract with the National Science Foundation; at the European Southern Observatory, La Silla, Chile; and at the Observatorio del Roque de los Muchachos, La Palma, Canary Islands. spots" in the lobes of radio galaxies. They are also expected to show synchrotron continua; their observation in the optical has the advantage of not being lost against the starlight near galactic nuclei such as hampers study of smaller scale jets, since hot spots occur many kiloparsecs from the nuclei. Several hot spots have been convincingly identified by position, color, or polarization. Saslaw, Tyson, and Crane (1978) reported faint optical objects in four radio hot spots, and later reported an additional nine (Crane, Tyson, and Saslaw 1983). Most of these objects are quite faint $(V=23)$, so further work is difficult. The same is true of the object in the southwest lobe of 3C 33 reported by Simkin (1978), though dedicated observational efforts have led to continuum shape, possible broad emission lines, and polarization measurements for this object (Meisenheimer and Röser 1986; Simkin 1986). A much brighter, highly polarized object has recently been found in the western hot spot of Pictor A (Röser and Meisenheimer 1987), in which the continuum is optically well observed, featureless, and flat, forming an exact continuation of the radio spectrum.

To extend our picture of the high-energy particle content of jets and hot spots, and to allow a more meaningful comparison of these classes, deep images have been obtained in several optical passbands for objects known to show optical radiation, or for which radio data suggested that the optical continuum might be detectable with currently available equipment. The data have been analyzed with particular attention to removal of the underlying starlight and nuclear light of each galaxy, the absolute calibration of the data, and in some cases image reconstruction to slightly increase the effective resolution and allow measurement of closely spaced features. The contribution of line emission, where observed, was evaluated and removed. The continuum shapes measured have been compared to simple synchrotron models and used to derive maximum electron energies produced in the jets under simple assumptions about local conditions; regularities among various objects suggest that characteristic scales are important 
in particle acceleration in kiloparsec-scale jets. In M87, systematic variations in the optical continuum slope are seen along the jet, implying some coupling between local conditions and position in the source.

An Appendix includes information on the surface brightness and color profiles of program galaxies, and on their environments.

\section{OBSERVATIONS AND DATA ANALYSIS}

\section{a) Galaxy Selection}

The primary sample for this program was intended to include those galaxies which either had known optical jets corresponding to the radio structures, or have radio morphology and surface brightness suggesting that optical continua might be observable. In the first category are M87, 3C 31 and 3C 66B (Butcher, van Breugel, and Miley 1980), 3C 277.3 = Coma A (van Breugel et al. 1985), 3C 273, and PKS $0521-36$ (e.g., Keel 1986). Objects of the second kind were found by examining radio maps of galaxies listed by Bridle and Perley (1984) as having probable radio jets. Several of these had in fact been observed by Butcher, van Breugel, and Miley (1980) with negative results, but instrumental improvements warranted a new examination. The criteria for inclusion of a radio galaxy (or low-redshift QSO) were roughly

1. apparent jet length in the range $4 "-10^{\prime \prime}$,

2. redshift below about 0.1 , and

3. jet flux greater than about $0.2 \mathrm{Jy}$ at $5 \mathrm{GHz}$.

Time constraints prevented observation of all objects in these categories. A few others (Cen A, Pic A) were observed primarily for different purposes and the data analyzed for this study. Overall properties of the galaxies (and two quasars) actually observed appear in Table 1 . Further details on individual systems appear in $\S$ IV.

Galactic reddening for each system was estimated following Burstein and Heiles (1984); for several objects it is negligible. Magnitudes quoted in the tables are as observed; flux densities (mJy) shown in the figures and used in model fitting have been corrected for galactic reddening.

\section{b) Images}

Images in a variety of passbands were obtained for the objects in Table 1 , using CCD cameras at several observatories. Since the structure in any synchrotron spectrum occurs only over a very broad frequency range, the full wavelength baseline from $B$ to $I$ was normally observed (few CCDs being sufficiently sensitive at shorter wavelengths); in a few cases, Gunn $z$, at about $0.95 \mu \mathrm{m}$, is available as well. The relatively high quantum efficiency of CCDs, their stability, and linearity are all important for this application, since jets generally appear as "perturbations" on galaxy isophotes at levels below $1 \%$. Table 2 lists the individual observations analyzed here. Each telescope is identified by its aperture in meters; the properties of CCDs used at various observatories are compared in Table 3.

The KPNO $2.1 \mathrm{~m}$ data, taken with a TI CCD having $15 \mu \mathrm{m}$ $\left(00^{\prime \prime}\right)$ pixels, are particularly suited for objects in which careful allowance for seeing or blending, or deconvolution of seeing effects, are important. The seeing at Kitt Peak was generally 1".0-1".3 FWHM. Filters at KPNO and CTIO were Mould $B V R I$ sets; a few images through $70 \AA$ FWHM filters centered on emission-line wavelengths were used to measure the contribution of [O III] $\lambda 5007$ or $\mathrm{H} \alpha+[\mathrm{N} \mathrm{II}] \lambda \lambda 6548,6583$ when emission lines were suspected.

The CTIO I images suffer from relatively severe fringing due to strong, time-variable night-sky $\mathrm{OH}$ emission. Its effects on the image of PKS $0521-36$ were reduced by taking a sequence of exposures with the telescope moved slightly in between, and adding reduced images in a coordinate system fixed on the sky. Bias-subtraction and flat fielding were normal; care was taken to keep statistical errors in the flat field frames well below $1 \%$ per pixel.

\section{c) Galaxy Subtraction and Jet Measurement}

For most of these objects, measurement of optical fluxes for the jets requires very accurate modeling and subtraction of the galaxy light distribution. Theoretical light profiles (Butcher, van Breugel, and Miley 1980) and reflection of the observed galaxy (Keel 1986) have been used for this; each suffers from disadvantages. As Cayette and Sol (1987) point out, the latter procedure can degrade the jet signal-to-noise ratio by a factor of $2^{1 / 2}$ per pixel, and some kind of galaxy modeling is preferable. Such an approach has been used here, based on the GRASP (or GASP) surface-photometry software, written by M. Cawson, as extended by R. Jedrzewski and further by $\mathbf{M}$. Franx and the author. The profile-determining routine of this system fits ellipses to the isophotes at various major axis radii, and the whole family of ellipses so generated is taken as a model of the galaxy profile, a fuller description is given by

TABLE 1

Properties of Program Galaxies

\begin{tabular}{|c|c|c|c|c|}
\hline Galaxy & Designation & Redshift & $B(t)$ & Notes \\
\hline M87 & $1228+12$ & 0.004 & 9.6 & NGC 4486 \\
\hline PKS ... & $0521-36$ & 0.055 & 15.4 & BL Lac nucleus \\
\hline 3C $31 \ldots \ldots \ldots \ldots \ldots$ & $0104+32$ & 0.0163 & 13.1 & \\
\hline 3C 66B $\ldots \ldots \ldots \ldots \ldots$ & $0220+42$ & 0.0215 & 13.8 & \\
\hline 3C $219 \quad \ldots \ldots \ldots \ldots \ldots$ & $0917+45$ & 0.1744 & 18.0 & \\
\hline 3C $273 \ldots$ & $1226+02$ & 0.158 & 12.8 & Quasar \\
\hline 3C $277.3 \ldots \ldots \ldots \ldots$ & $1251+27$ & 0.0857 & 16.7 & Coma A \\
\hline 3C $296 \ldots \ldots \ldots \ldots \ldots$ & $1414+11$ & 0.0237 & 13.1 & NGC 5532 \\
\hline 3C $303 \ldots \ldots \ldots \ldots$ & $1441+52$ & 0.1410 & 17.5 & Optical hot spot \\
\hline 3C $449 \quad \ldots$. & $2229+39$ & 0.0171 & 14.0 & Twin-jet source \\
\hline NGC $6251 \ldots$ & $1638+82$ & 0.0234 & 14.8 & \\
\hline B2 $\ldots \ldots \ldots \ldots$ & $1321+31$ & 0.0161 & 14.6 & \\
\hline Pictor $\mathrm{A} \ldots \ldots \ldots \ldots \ldots$ & $0518-45$ & 0.0350 & 16.5 & \\
\hline PKS & $0812+02$ & 0.402 & 18.5 & Quasar, 4C 02.23 \\
\hline Cen A & $1322-42$ & 0.001 & 8.0 & NGC 5128 \\
\hline
\end{tabular}


TABLE 2

JOURNAL OF OBSERVATIONS

\begin{tabular}{|c|c|c|c|c|c|}
\hline Object & Telescope & Passband & Exposure (min) & U.T. Date & Remarks \\
\hline \multirow[t]{6}{*}{ M87 } & 2.1 & $B$ & 20 & 1985 May 23 & Clouds \\
\hline & & $V$ & 10 & 1985 May 23 & Clouds? \\
\hline & & $R$ & 10 & 1985 May 23 & \\
\hline & & & 10 & 1985 May 23 & \\
\hline & 2.2 & $\boldsymbol{V}$ & 15 & 1986 Apr 7 & Large airmass \\
\hline & & $z$ & 5 & 1986 Apr 7 & Large airmass \\
\hline \multirow[t]{2}{*}{ 3C $31 \ldots \ldots \ldots \ldots \ldots \ldots$} & $2.1^{*}$ & $V$ & 16 & 1986 Sep 26 & Clouds \\
\hline & & $R$ & 16 & 1986 Sep 26 & Clouds \\
\hline \multirow[t]{3}{*}{ 3C 66B $\ldots \ldots \ldots \ldots \ldots \ldots$} & $2.1^{*}$ & $V$ & 16 & 1986 Sep 26 & Clouds \\
\hline & & $R$ & 16 & 1986 Sep 26 & Clouds \\
\hline & 2.5 & B & 45 & 1987 Feb 23 & \\
\hline \multirow[t]{2}{*}{$3 C 219$} & 2.1 & $B$ & 40 & 1985 May 25 & \\
\hline & & $R$ & 25 & 1985 May 25 & \\
\hline \multirow[t]{4}{*}{ 3C 273} & 2.1 & $B$ & 12 & 1985 May 23 & \\
\hline & & $\boldsymbol{V}$ & 20 & 1985 May 25 & \\
\hline & & $R$ & 25 & 1985 May 25 & \\
\hline & & $I$ & 17 & 1985 May 27 & \\
\hline \multirow{5}{*}{$\begin{array}{c}3 \text { C } 277.3 \ldots \\
(\text { Coma A) }\end{array}$} & 2.1 & $B$ & 40 & 1985 May 24 & \\
\hline & & $V$ & 20 & 1985 May 24 & \\
\hline & & $R$ & 20 & 1985 May 24 & \\
\hline & & $I$ & 25 & 1985 May 24 & \\
\hline & & $\lambda 7146$ & 35 & 1985 May 24 & $\mathrm{H} \alpha+[\mathrm{N} \mathrm{II}]$ \\
\hline \multirow[t]{2}{*}{ 3C $296 \ldots \ldots \ldots \ldots \ldots$} & 2.1 & B & 60 & 1985 May 25 & \\
\hline & & $R$ & 25 & 1985 May 25 & \\
\hline \multirow[t]{4}{*}{ 3C $303 \quad \ldots \ldots \ldots \ldots \ldots \ldots$} & 2.1 & $B$ & 40 & 1985 May 24 & \\
\hline & & $V$ & 20 & 1985 May 24 & \\
\hline & & $\boldsymbol{R}$ & 20 & 1985 May 24 & \\
\hline & & $I$ & 25 & 1985 May 24 & \\
\hline 3С $449 \quad \ldots \ldots \ldots \ldots \ldots$ & 2.1 & $B$ & 60 & 1985 May 25 & \\
\hline \multirow{2}{*}{$\begin{array}{l}\text { NGC } 5127 \ldots \ldots \ldots \ldots \\
\quad(B 21321+21)\end{array}$} & 2.1 & $B$ & 30 & 1985 May 23 & \\
\hline & & $V$ & 30 & 1985 May 23 & \\
\hline \multirow{7}{*}{$\begin{array}{l}\text { NGC } 5128 \ldots \ldots \ldots \ldots . . \\
(\text { Cen A) }\end{array}$} & 1.5 & $B$ & 10 & 1986 Apr 16 & \\
\hline & & $\boldsymbol{V}$ & 6 & 1986 Apr 16 & \\
\hline & & $R$ & 5 & 1986 Apr 16 & \\
\hline & & $I$ & 5 & 1986 Apr 16 & \\
\hline & & $\lambda 6563$ & 15 & 1986 Apr 16 & \\
\hline & 2.2 & $V$ & 10 & 1986 Apr 17 & \\
\hline & & $z$ & 20 & 1986 Apr 17 & \\
\hline \multirow[t]{4}{*}{ NGC $6251 \ldots \ldots \ldots \ldots$} & 2.1 & $B$ & 60 & 1985 May 23 & \\
\hline & & $B$ & 60 & 1985 May 24 & \\
\hline & & $\boldsymbol{V}$ & 40 & 1985 May 23 & \\
\hline & & $R$ & 40 & 1985 May 28 & \\
\hline \multirow[t]{6}{*}{ PKS $0521-36 \ldots \ldots \ldots$} & 4.0 & $B$ & 10 & 1983 Sep 29 & \\
\hline & & $V$ & 8 & 1983 Sep 29 & \\
\hline & 3.6 & $\boldsymbol{R}$ & 3 & 1986 Apr 4 & \\
\hline & 1.5 & $R$ & 20 & 1986 Apr 14 & \\
\hline & & $I$ & 20 & 1986 Apr 14 & $2 \times 10 \min$ \\
\hline & & $I$ & 20 & 1986 Apr 16 & $4 \times 5 \min$ \\
\hline \multirow[t]{2}{*}{ PKS $0821+02 \ldots \ldots}$. & 1.5 & B & 10 & 1986 Apr 14 & \\
\hline & & $\lambda 7007$ & 30 & 1986 Apr 14 & [O III] \\
\hline \multirow{2}{*}{ Pictor A ............... } & 1.5 & $V$ & 15 & 1986 Apr 15 & \\
\hline & & $R$ & 15 & 1986 Apr 15 & \\
\hline
\end{tabular}

TABLE 3

IMAGING CONFIGURATIONS

\begin{tabular}{|c|c|c|c|c|c|c|}
\hline $\begin{array}{c}\text { Telescope } \\
\text { Aperture (m) }\end{array}$ & Observatory & CCD & Format & $\begin{array}{c}\text { Scale } \\
\text { ("per pixel) }\end{array}$ & $\begin{array}{l}\text { Readout Noise } \\
\text { (electrons) }\end{array}$ & Filters \\
\hline 1.5 & CTIO & RCA\#5 & $354 \times 512$ & 0.54 & 40 & $B V R I, \mathrm{H} \alpha$ \\
\hline $2.1 \ldots \ldots \ldots \ldots$ & KPNO & TI \#2 & $800 \times 800$ & 0.19 & 16 & $B V R I, \mathrm{H} \alpha$ \\
\hline $2.1^{*} \ldots \ldots \ldots \ldots \ldots$ & KPNO & RCA\#1 & $320 \times 512$ & 0.38 & 75 & $V R$ \\
\hline $2.2 \ldots \ldots \ldots \ldots$ & ESO & RCA & $320 \times 512$ & 0.35 & 40 & $V R I Z$ \\
\hline $2.5 \quad \ldots \ldots \ldots \ldots \ldots$ & INT, La Palma & RCA & $320 \times 512$ & 0.83 & 63 & $B$ \\
\hline $3.6 \ldots \ldots \ldots \ldots$ & ESO & RCA & $320 \times 512$ & 0.675 & 45 & $R$ \\
\hline $4.0 \ldots \ldots+\cdots$ & CTIO & RCA & $320 \times 512$ & 0.60 & 90 & $B V$ \\
\hline
\end{tabular}


Davis et al. (1985). Portions of each image affected by blemishes, stars, other galaxies, globular clusters (in M87), or expected jet emission were excluded from the regions to be fitted in some cases iteratively. Once a satisfactory profile (consisting of a set of intensity values; semimajor axes, ellipticities, position angles, and center coordinates) had been fitted to each image, a model galaxy image was generated by interpolation between these (using the RJSIMGAL routine by Jedrzewski). A slight modification of the program was needed to correct the interpolation of position angle in nearly round galaxies where the formal P.A. swings by $180^{\circ}$ between successive isophotes, but in which the real P.A. has changed by only a few degrees. The model was then subtracted from the data, leaving a residual image including stars, faint galaxies, and in many cases emission from the jets. If objects appeared in the difference frames that might affect isophote measurement and had not been apparent in the original data (due to strong galaxy background), a new mask was generated and the image remeasured.

Most galaxies were measured with a starting isophote radius of one pixel, increasing by a factor of 1.1 per step. Measurement proceeded until less than half an isophote was included in the data, or the intensity per pixel dropped below two image counts (ADUs) above the sky background. Program limitations allowed only a $512 \times 512$ area to be treated at once; larger data frames were either binned $2 \times 2$ or the central portions alone measured (or both). For the largest galaxies, the background found from the edge regions will not be actual blank sky, but contain a contribution from the outer parts of the galaxies. This does not affect measurement of the jet emission, but certain surface-photometry results (Appendix A) have been corrected using other data in order to reduce the bias so introduced.

Several of the program galaxies have isophotes that are significantly "boxy" in the region of interest. It has in fact been suggested (Bender and Möllenhoff 1987) that boxy isophotes and strong radio emission are statistically connected. The residuals from a simple elliptical fit are as much as a few percent of the original intensity in the worst cases, seriously reducing the detectability of faint features (particularly in NGC 6251 , in which a corner of the "box" is aligned with the jet). This was treated by measuring the best-fitting constants in the expression for residuals from an elliptical model

$$
I(\Theta)=I_{0}+A \cos 4\left(\Theta-\Theta_{0}\right),
$$

evaluated on a circle about the center of each galaxy. The amplitude changes slowly enough with radius that the refinement of tracing these points along the elliptical isophotes was unnecessary. This fit was performed with masking of stars and jets, represented as azimuthal sectors.

This two-step modeling was very successful in removing the galaxy light from the data. In galaxies without very strong boxiness, the residual noise after model subtraction was as expected for Poisson noise from the original intensity level for each point in the image. Some detectable systematic residuals from boxy isophotes appear in the "boxiest" systems, such as 3C 296, indicating that the simple fourth-order treatment here is not entirely correct, though adequate for these purposes. Figure 1 (Plate 13) is a high-contrast display of M87, showing the high accuracy to which galaxy light is removed (to better than $0.1 \%$ in the inner parts). Figure 2 (Plate 14) shows images of $3 \mathrm{C} 66 \mathrm{~B}$ and NGC 6251 in which jet emission appears.
Figure 3 (Plate 15) shows the knots in Coma A and 3C 303 (which did not require galaxy subtraction).

The GRASP routines deal only with integer data. In a few instances, involving the outer parts of galaxies observed with a low-noise chip, this leads to a residual image showing slight ripples (since the areas concerned cover many pixels, sub-ADU changes may be discerned). This was countered, where needed, by scaling the data by a factor 10 before measurement.

Wherever possible, a star image near each galaxy was measured to provide an estimate of the point-spread function (PSF). This was usually measured after subtracting the galaxy background. Thus, the seeing profile is accurately known for each image; this is particularly important for cases in which marginally resolved substructure is present, as well as for evaluation of the surface photometry in distant systems.

Having obtained images of radio jets free of contamination by the galaxies' starlight (except for increased Poisson noise), and with the PSF well determined, several strategies are available for measuring the optical intensity of a jet. Straightforward photometry--summing detected counts in a circle, box, or more specific shape-suffices for the brightest objects in the sample, the errors being dominated by uncertainties in the photometric zero points. For fainter objects more elaborate processing may be required.

As a first step in detecting emission with intensities below the pixel-to-pixel background noise, the well-sampled TI 2 images were convolved with a Gaussian somewhat smaller than the seeing disk (for example, 0"7 FWHM in 1".0 seeing). This gain (a factor 3-4 in limiting surface brightness) is enough to show, for example, the brightest knot in the jet of NGC 6251. In these cases, the optical detections stand on their own, and measurement is again rather straightforward, with some guidance from the radio data.

Some galaxies show no optical emission from the jets even after smoothing. Limits in these cases were derived from smoothed residual images, in the regions of strongest radio emission.

\section{d) Calibration}

On photometric nights, the magnitude (flux) scale was set by observations of standard stars from the list of Christian et al. (1985). Mean extinction coefficients were used due to the usually small airmass range for standards. For objects observed through clouds, and for some others as a check, the zero points were set by comparison with aperture photometry or published surface photometry. Aperture photometry was taken from the compilation by Longo and de Vaucouleurs (1983), as well as new $U B V R I$ observations obtained in 1987 February with the $1 \mathrm{~m}$ Kapteyn telescope on La Palma (Table 4). Note that published magnitudes scatter by about 0.05 even in bright galaxies at a given aperture size, perhaps due to uncertainties in exact aperture diameter or centering, so that a large number of measures may be needed for accurate comparison with CCD data. Surface photometry was taken from the work of Davis et al. (1985) for M87 and Young et al. (1979) for NGC 6251, with a mean correction from $r$ to $R$ magnitudes. For data taken under photometric conditions the zero-point difference between the present data and published surface photometry is within the errors set by uncertainty in the exact image scale for the new images (about $4 \%$ in surface brightness, not applicable to total intensities of objects).

For conversion of the $B V R I$ data into energy units (flux densities), the zero points given by Johnson (1966) were used. 
TABLE 4

Photoelectric Aperture Photometry

\begin{tabular}{ccccccc}
\hline \hline Galaxy & $\begin{array}{c}\text { Aperture } \\
(\text { (") }\end{array}$ & $V$ & $B-V$ & $U-B$ & $V-R$ & $V-I$ \\
\hline 3C 66B $\ldots \ldots \ldots \ldots$ & 21 & 14.01 & 1.03 & 0.63 & 0.69 & 0.83 \\
& & \pm 0.02 & 0.02 & 0.03 & 0.01 & 0.03 \\
Coma A .......... & \multirow{2}{*}{14} & 16.64 & 1.14 & 0.70 & 0.54 & 1.22 \\
& & \pm 0.05 & 0.02 & 0.05 & 0.05 & 0.04 \\
\hline
\end{tabular}

The mean wavelengths quoted for jet emission incorporate the actual filter and CCD responses, the measured spectral shape of the jet when seen, and the galaxy redshift (all are quoted in the galaxies' rest frames). In some cases, the absolute calibration uncertainty limits the detail with which models may be fitted to the broad-band data.

\section{e) Image Restoration}

The M87 jet images indicate that there is important structure at and just below the seeing scale, as is seen in the radio (Biretta, Owen, and Hardee 1983). The complex radio structure suggested that some level of modeling would be required to evaluate the spectral shapes of even the most prominent knots in the jet. The radio structure and the present results also imply that modeling as a sum of point sources or Gaussians convolved with seeing is probably inadequate for these purposes. Hence, a direct determination of the sub-arcsecond optical structure was sought.

Data of high signal-to-noise ratio, if well-enough sampled across the seeing profile and within the detector's linear range, can in principle be processed in several ways to yield a modest (factor 2) increase in linear resolution, at the expense of local noise. Several algorithms are available for this. Maximumentropy reconstruction (recently reviewed by Narayan and Nityananda 1986) is appealing from an information-theoretic standpoint but has serious drawbacks in measuring colors of small regions; resolution in the restored image is a function of input intensity level, and the flux of image subsections is not conserved. Similar objections apply to the pixel-compensation techniques applied to images of M87 by Lorre and Nieto (1984). I have instead used a modified form of the CLEAN algorithm often used to remove sidelobes in radio aperturesynthesis maps (see Clark 1980). The method proceeds by subtracting scaled versions of the PSF from successive peaks in the residual image, for as long as significant flux remains to be accounted for. This yields a model of the image as a set of point sources (one per pixel in the limit of an extended source), usually convolved with a "restoring beam" of known properties before analysis.

Minor modifications to the standard CLEAN are needed for efficient work on optical images. The noise in aperturesynthesis maps is fundamentally in the Fourier domain, while in optical images it exists in the image plane. Thus there is structure due to pixel-to-pixel Poisson noise on scales finer than could be produced by any positive image after convolution with the PSF. Normal application of CLEAN, upon encountering the highest noise peak, will generate pairs of increasingly positive and negative components to match this spurious fine-scale structure, to the exclusion of anything else in the image. An acceptable remedy, suggested by conversations with W. Jaffe, is smoothing the current residual image before finding the peak value for PSF subtraction. In practice, a smoothing size close to the seeing-profile FWHM was found suitable. This reduced "noise-locking" sufficiently to allow cleaning to less than $1 \%$ of the jet's peak intensity, and until the total flux found by CLEAN converged.

Evaluation of a proper PSF was complicated by the lack of bright stars (above magnitude 21 at $V$ ) near M87. The brightest object within $1: 2$, at $V=21$, is resolved at the 0 .'5 level and is perhaps a member of the background cluster of galaxies found by Huchra and Brodie (1984) at $z=0.086$, or an even more distant object. Genuine globular clusters in the field, while certainly unresolved enough for a measurement of the PSF, are all too faint for even a parameterized determination. The PSF was finally taken from stars in the nearby 3 C 273 field observed immediately after M87. Tests were made using CLEAN and the PSF scaled by $10 \%$ up and down in size, to see whether seeing changes would have important effects on the results. None of the conclusions here depends critically on the parameters of particular CLEAN runs or on the PSF size to within $10 \%$; the latter is due to the effective resolution limit of 0 ." 4 set by the 0.2 pixels.

The efficacy of this technique may be judged from Figure 4 (Plate 16), which compares observed and cleaned $B$-band images of the M87 jet, together with a star image. The optical structure matches that seen at $2 \mathrm{~cm}$ (Biretta, Owen, and Hardee 1983) very well indeed; the structure must match at better than the 0.4 level to achieve this detailed agreement. Similar processing was done for the $V, R$, and $I$ images; the same structures could be identified in each case, and fluxes were measured for exactly matching regions in each cleaned image. The final signal-to-noise ratios in each passband are similar, since the exposure times were chosen to give similar numbers of total counts in the jet. Further analysis of these data appears in $\S$ III.

\section{f) Spectroscopy}

It is important to verify that the objects studied are in fact continuum sources, without unrecognized strong line emission that could compromise broad-band measurements. This has been done in detail for the M87 jet (see $\S$ III $a$ ), and sufficiently for 3C 273 to confirm that no strong lines are present. I have examined the jet of PKS 0521 - 36 spectroscopically, confirming its continuum nature and the steep slope found from images.

The ESO faint object spectrograph and camera (EFOSC; D'Odorico and Dekker 1986) was used at the $3.6 \mathrm{~m}$ telescope. A total of 30 minutes exposure was obtained, covering the range $3800-7000 \AA$ with useful sensitivity. The slit was aligned along the jet in P.A. $302^{\circ}, 35^{\circ}$ from the zenith direction. Over the air mass range covered by the observations, the mean component of atmospheric dispersion between 3800 and $7000 \AA$ perpendicular to the slit was only 0".65 (following Filippenko 1982). For a 1".5 slit centered on the jet, this will have notice- 
able effects only at the extreme blue end of the spectrum. Flux calibration used the standard stars Feige 56 and LTT 7987 at similar zenith distances.

Scattered light from the nucleus and underlying starlight were removed by reflecting the spectrum about the central continuum source. The residual light at the jet position is quite weak in comparison with the nucleus, but at the correct level obtained from image analysis (Fig. 5). The spectrum of the whole jet, smoothed, is shown in Figure 6. The slope of the continuum is consistent with determinations from images, and no believable spectral features are present. The red knot at the tip (Danziger et al. 1979; Keel 1986) was detected as a continuum source longward of about $6000 \AA$, but no stronger statement is possible from these data. Because of uncertainties in the (large) scattered-light correction, no separation between the inner strong knot and the rest of the jet is presented.

Higher resolution observations of the $3 \mathrm{C} 66 \mathrm{~B}$ jet in the $\mathrm{H} \alpha$ region were conducted at the KPNO $4 \mathrm{~m}$, with a TI CCD on the Ritchey-Chrétien spectrograph. An exposure of $3000 \mathrm{~s}$ duration at a resolution of $2.5 \AA$ was obtained. From these data it is clear that emission in lines from 6300 to $6800 \AA$ is not a significant contribution to the jet flux in this passband, so this jet is also a (pure) continuum source.

\section{SPECTRAL CHANGES ALONG THE M87 JET}

\section{a) Previous Work}

The jet of M87 has long been known to shine primarily by synchrotron radiation. Its overall optical spectrum is contin- uous (Baade and Minkowski 1954; Sulentic, Arp, and Lorre 1979; Keel 1984), and the knots show high polarization (Baade 1956; Schmidt, Peterson, and Beaver 1978). The optical and UV spectral shape is certainly close to a power law, but is clearly steeper than that observed at centimeter wavelengths (Pronik, Pronik, and Chuvaev 1968; Perola and Tarenghi 1980). A spectral break has been inferred at various wavelengths between 0.3 and $1.0 \mu \mathrm{m}$ (Kinman, Grasdalen, and Rieke 1974; Stocke, Rieke, and Lebofsky 1981; Keel 1984), which must be similar for all parts of the jet whatever its exact value. The differences among spectral slopes determined in various ways have been uncomfortably large, most likely due to the strong background light against which the jet is seen.

The jet structure at the $1^{\prime \prime}$ scale is dominated by a series of small knots, with at most weak evidence for continuous emission between them (de Vaucouleurs, Angione, and Fraser 1968; de Vaucouleurs and Nieto 1979; Arp and Lorre 1976; Nieto and Leievre 1982; Lorre and Nieto 1984). The morphology in the optical matches that seen with similar resolution in the radio, suggesting a dominant role for magnetic field configuration in producing the observed structure (Nieto and Lelievre 1982; Shklovskii 1984; Keel 1984; Lorre and Nieto 1984; see $\S$ III $c$ ).

There have been several reports of variability in flux or polarization of individual knots, or of the jet as a whole (Pronik and Shcherbalov 1972; Sulentic, Arp, and Lorre 1979; Warren-Smith, King, and Scarrott 1984). The complex structure of the jet and the resulting interplay with resolution effects make such measurements difficult, if potentially important.

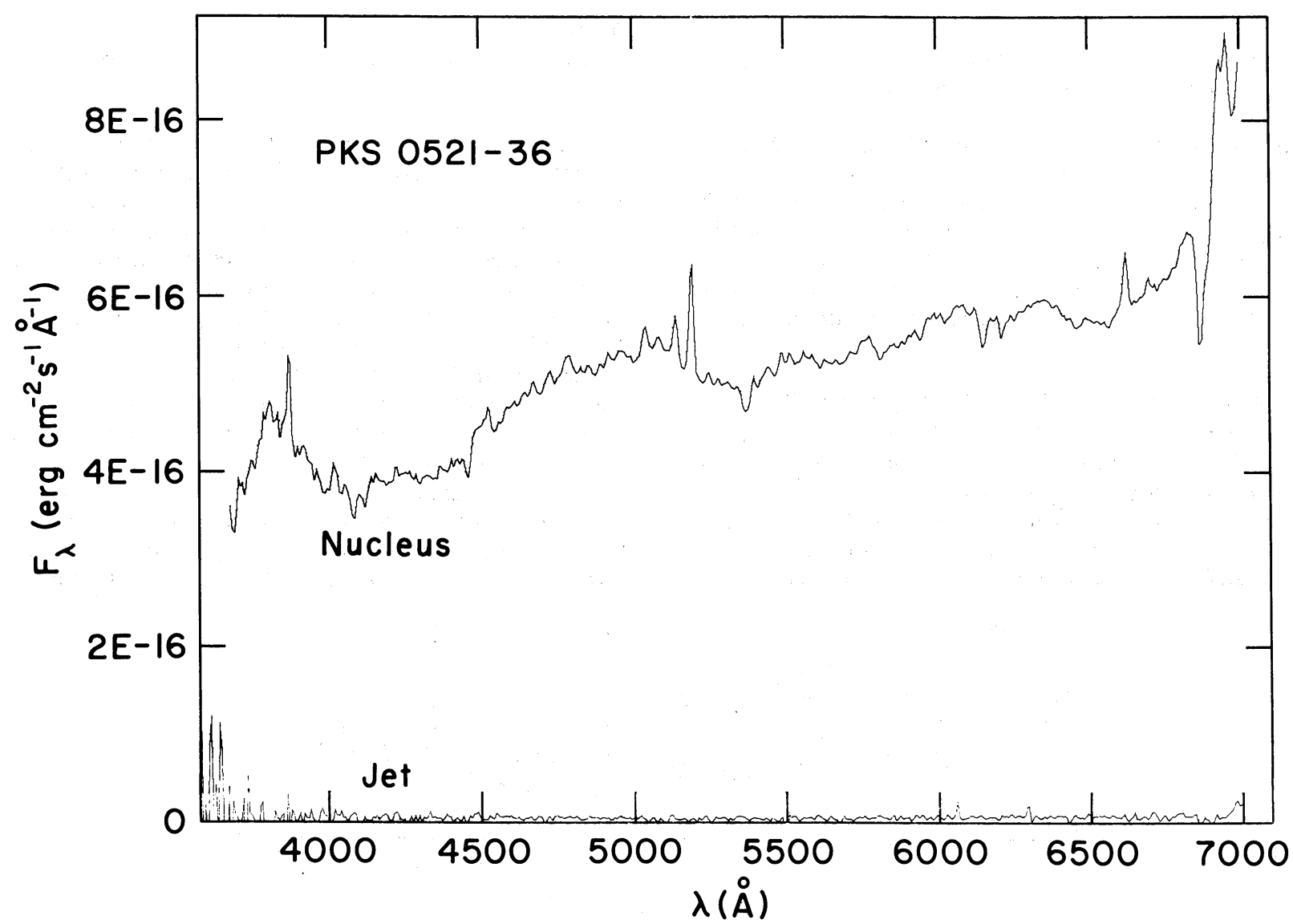

FIG. 5.-EFOSC spectra of the nucleus and jet of PKS 0521 - 36, shown on a common scale. Scattered nuclear light and galaxy background have been removed at the jet position by assuming symmetry along the slit. 


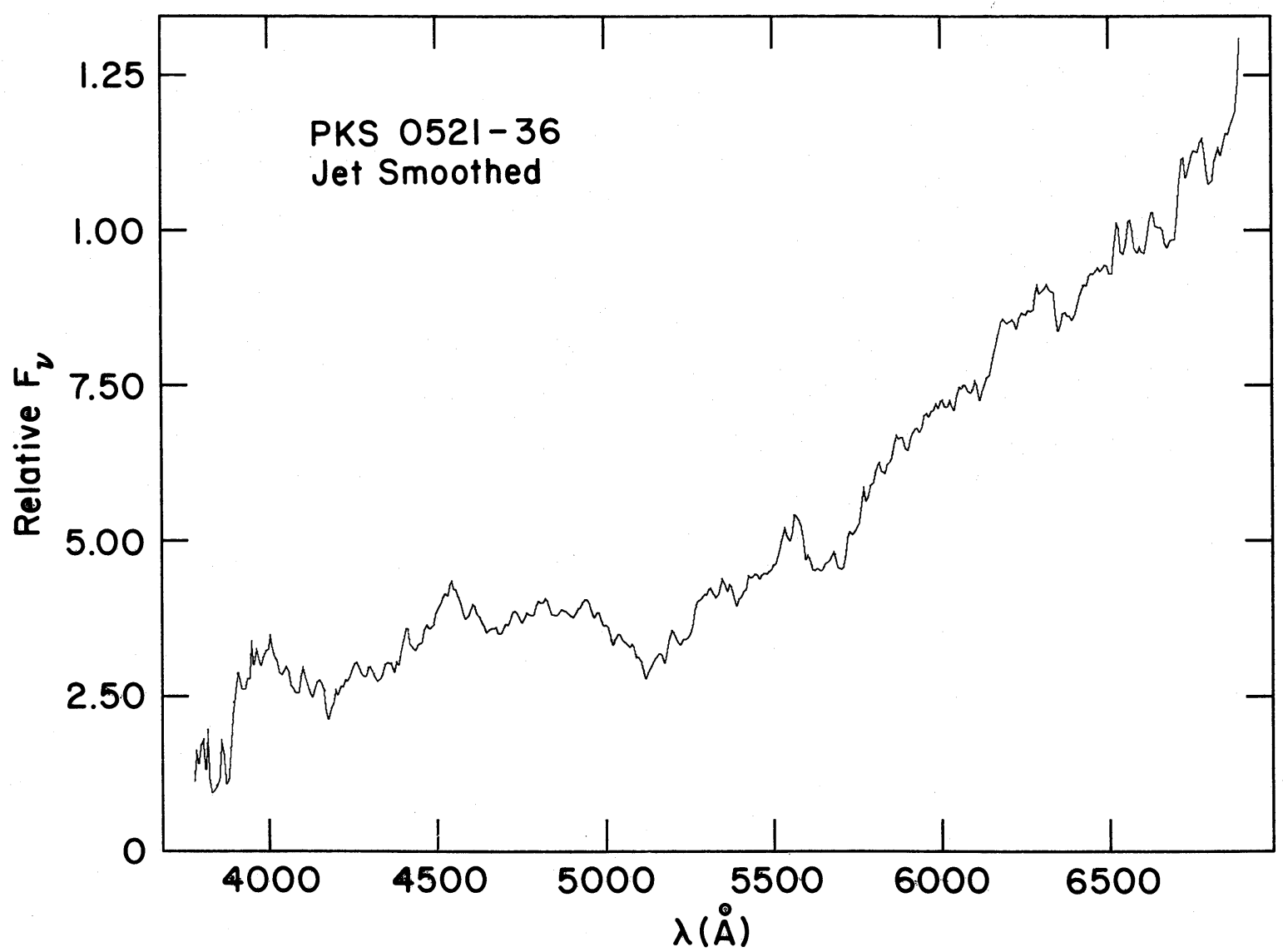

Fig. 6. -EFOSC spectrum of the jet of PKS $0521-36$, smoothed with a $125 \AA$ rectangular filter and shown as flux per unit frequency. The steep continuum matches that seen from multicolor imaging; no significant spectral features are present.

Several important uncertainties remain from these studiesin particular, just what is the shape of the optical continuum, and does this change along the jet? While M87 will certainly be an early Space Telescope target, techniques are now available allowing a more precise set of measures than hitherto reported. Particular care was given to the M87 jet in this study, in an effort to resolve some of the discrepancies among earlier studies.

\section{b) Deconvolved Images and Knot Measurements}

The galaxy-removal procedure used here allows a direct evaluation of how accurately starlight has been removed from the jet, which has been a major potential source of error in many studies. As may be seen from Figure 1, any residual galaxy light near the jet must be very small; it cannot systematically exceed $0.1 \%$ of the original intensity near the nucleus.
The complexity of the radio structure seen at high resolution (Biretta, Owen, and Hardee 1983) suggested that modeling with a small set of symmetric components would be misleading, so the CLEAN approach described in $\S$ II $e$ was adopted. This is in essence a modeling procedure driven to whatever complexity is supported by the data. The close resemblance between the results (Fig. 4) and Figure 1 of Biretta, Owen, and Hardee indicates that the reconstruction is accurate.

Features in the jet were identified from the optical images (the same ones could be seen in each passband), and fluxes measured in each image by integration over the regions of pixels matching in position and area for each image. The measured features are identified in Figure 7, and the measurements listed in Table 5. The error estimates reflect Poisson errors from the jet knots and original background levels, and binning errors (i.e., half-pixel positional uncertainties from image to

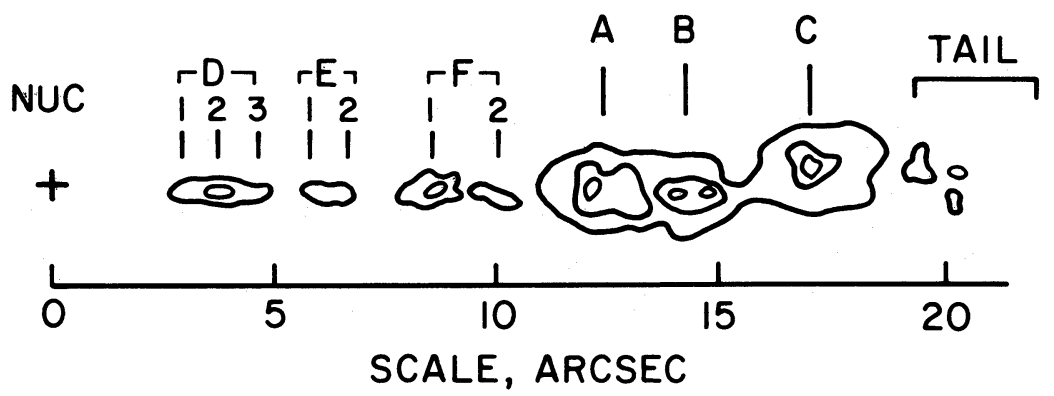

Fig. 7.-Contour display of cleaned M87 jet data, showing nomenclature and subdivisions of knots 
TABLE 5

Optical FluXes of Features in the M87 Jet

\begin{tabular}{|c|c|c|c|c|c|}
\hline \multirow{2}{*}{$\begin{array}{c}\text { FEATURE } \\
\text { (Jy) }\end{array}$} & \multicolumn{5}{|c|}{ FLUX (mJy) } \\
\hline & $B$ & $\boldsymbol{V}$ & $R$ & $I$ & $6 \mathrm{~cm}$ (Jy) \\
\hline $\mathbf{A}$ & $0.96 \pm 4$ & $1.08 \pm 3$ & $1.25 \pm 3$ & $1.48 \pm 4$ & 1.72 \\
\hline B $\ldots \ldots \ldots \ldots \ldots \ldots$ & $0.44 \pm 2$ & $0.59 \pm 2$ & $0.74 \pm 6$ & $0.80 \pm 2$ & 1.44 \\
\hline $\mathbf{B}^{\prime} \ldots \ldots \ldots \ldots \ldots$ & $0.08 \pm 1$ & $0.07 \pm 1$ & $0.06 \pm 1$ & $0.06 \pm 1$ & $(0.49)$ \\
\hline $\mathrm{C}, \ldots \ldots \ldots \ldots \ldots$ & $0.30 \pm 1$ & $0.40 \pm 2$ & $0.45 \pm 2$ & $0.59 \pm 2$ & 1.20 \\
\hline$D_{1} \ldots \ldots \ldots \ldots \ldots$ & $0.022 \pm 1$ & $0.036 \pm 3$ & $0.039 \pm 4$ & $\ldots$ & $\ldots$ \\
\hline $\mathrm{D}_{2} \ldots \ldots \ldots \ldots \ldots$ & $0.053 \pm 2$ & $0.057 \pm 3$ & $0.060 \pm 3$ & $0.097 \pm 2$ & $\ldots$ \\
\hline $\mathrm{D}_{3} \ldots \ldots \ldots \ldots \ldots$ & $0.025 \pm 1$ & $0.028 \pm 1$ & $0.029 \pm 1$ & $0.040 \pm 1$ & $\ldots$ \\
\hline $\mathrm{D}$ & $0.100 \pm 3$ & $0.121 \pm 2$ & $0.127 \pm 5$ & $(0.182) \pm 3$ & 0.22 \\
\hline$E_{1} \ldots$ & $0.056 \pm 2$ & $0.040 \pm 1$ & $0.045 \pm 1$ & $0.060 \pm 1$ & $\ldots$ \\
\hline$E_{2} \ldots$ & $0.019 \pm 1$ & $0.032 \pm 1$ & $0.035 \pm 3$ & $0.032 \pm 1$ & $\ldots$ \\
\hline $\mathrm{E}^{2} \ldots \ldots \ldots \ldots \ldots$ & $0.065 \pm 2$ & $0.073 \pm 2$ & $0.081 \pm 4$ & $0.092 \pm 1$ & 0.13 \\
\hline$F_{1} \ldots \ldots \ldots \ldots \ldots$ & $0.097 \pm 4$ & $0.104 \pm 10$ & $0.132 \pm 2$ & $0.164 \pm 2$ & $\ldots$ \\
\hline $\mathbf{F}_{2} \ldots$ & $0.027 \pm 1$ & $0.032 \pm 2$ & $0.050 \pm 1$ & $0.046 \pm 1$ & $\ldots$ \\
\hline $\mathbf{F} \ldots \ldots \ldots \ldots$ & $0.124 \pm 4$ & $0.136 \pm 4$ & $0.182 \pm 2$ & $0.210 \pm 2$ & 0.27 \\
\hline "Tail" ............ & $0.069 \pm 3$ & $0.074 \pm 2$ & $0.112 \pm 3$ & $0.104 \pm 1$ & $0.84:$ \\
\hline Total ............ & 1.96 & 2.40 & 2.81 & 3.42 & 5.82 \\
\hline
\end{tabular}

Errors are in units of the final significant figure listed.

image). Similar measurements are also listed at $6 \mathrm{~cm}$, from a 0 . 4 resolution map provided by $\mathrm{F}$. Owen.

As found by other workers ( $\S$ III $a$ ), the spectra are all very nearly power laws. The present data are plotted in Figure 8 , with indication of the levels expected by extrapolation of the

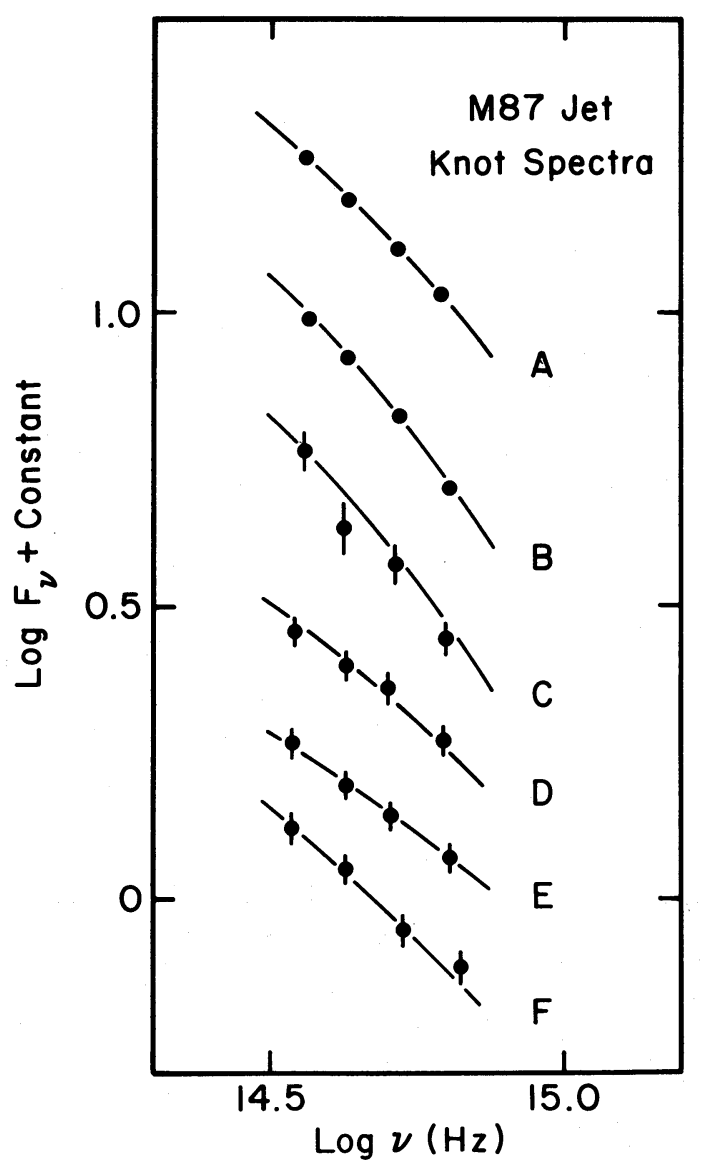

Fig. 8.-Spectra of individual knots in the M87 jet. The curves represent the best-fitting truncated synchrotron spectra, as in Table 7. radio continua. All knots have steeper optical than radio spectra; several show curvature in the optical.

A new result of this analysis is that the spectra of various knots are not exactly identical-there are significant changes along the jet. This is shown in Fig. 9, in which the mean optical spectral index (ignoring curvature for now) is plotted as a function of distance from the nucleus. The optical index steepens by about $\Delta \alpha=0.3$ going from the nucleus to the tip of the (optical) jet, with breaks in this trend being knots $\mathrm{A}$ and $\mathrm{E}$, which return to the slope seen near the nucleus. Only the highenergy part of the spectrum changes; the low-frequency index, determined between $6 \mathrm{~cm}$ and $0.8 \mu \mathrm{m}$, is in the range $-0.65 \pm 0.02$ for all the knots. Comparison with synchrotron models $(\S \mathrm{V})$ indicates that this is a change in cutoff frequency of the most energetic electrons, so either their energy or the local field strength is changing. It is, in any case, evidence of a connection between local (particle-acceleration) and global (position within the source) properties of the jet material. There are differences in the particle populations of otherwise similar regions at different locations in the jet.

The optical spectral-index changes along the jet bracket the range reported in the literature for the entire jet; different weightings along the jet may account for part of the scatter in published values.

\section{c) Optical Structure}

As has been noted by several workers ( $\S$ III $a$ ), the close match of the jet structure (knot positions, sizes, shapes) as seen at various wavelengths sets strong limits on how far the radiating electrons actually stream freely, either due to radiative losses or to tangled field structure which constrains the particles to more nearly closed paths. The most stringent limits have been set for the knot A region, for which the image restoration at $U$ and $B$ by Lorre and Nieto (1984) implies a match of $2 \mathrm{~cm}$ and $0.4 \mu \mathrm{m}$ structure at the 0.2 level. The present work extends this conclusion, at lower resolution, to the whole jet, especially the inner knots (D-I). Their structure, throughout the optical range, matches that at $2 \mathrm{~cm}$ at an effective resolution of 0.4 , so 0.3 is a conservative upper limit to any possible differential lifetime effect. For a distance of $20 \mathrm{Mpc}$, 0 ".3 corresponds to $30 \mathrm{pc}$. The synchrotron lifetime for par- 


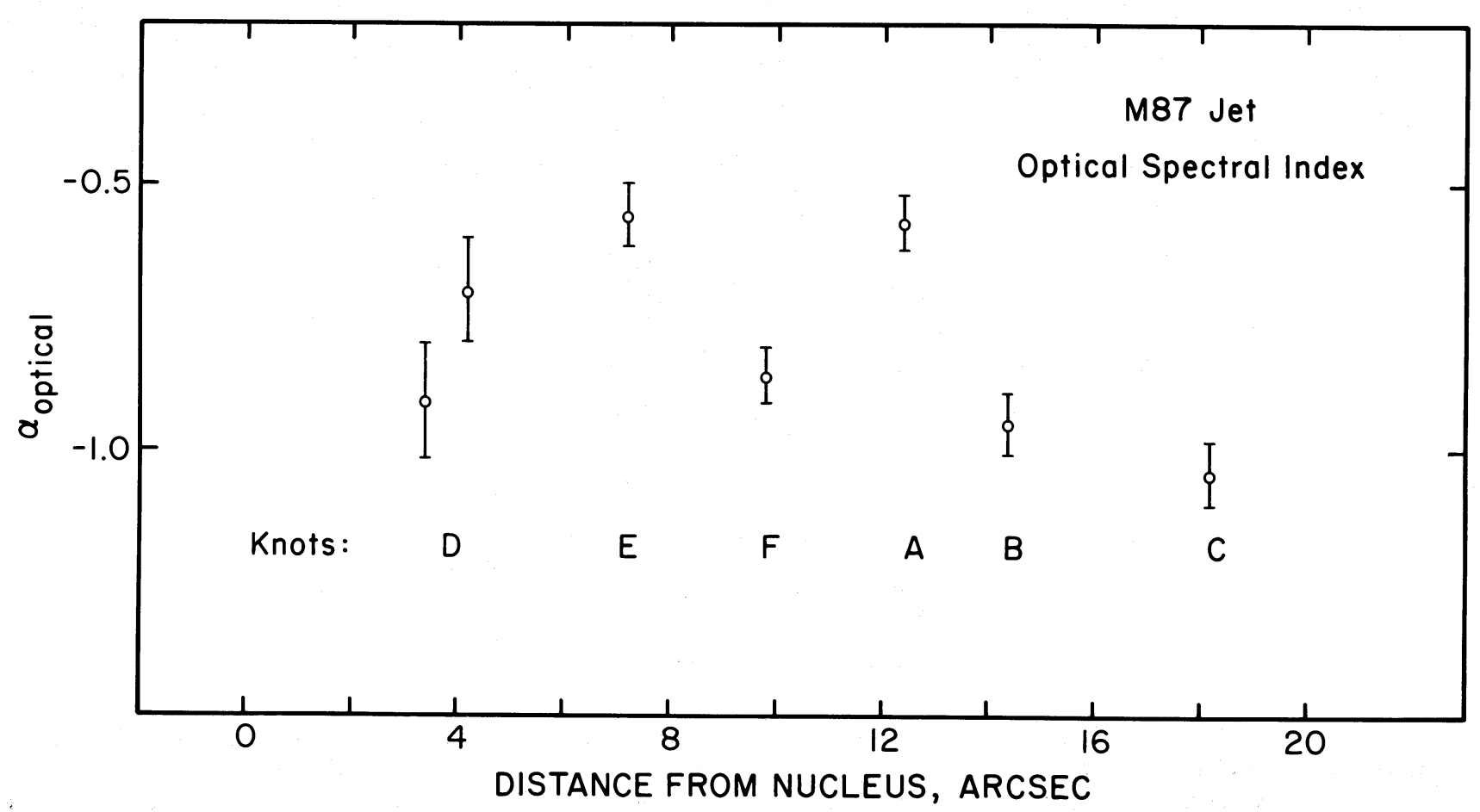

FIG. 9.-Mean optical spectral index $\alpha_{\text {optical }}$ as a function of position along the M87 jet. Locations of knot complexes are marked.

ticles radiating with a peak frequency $v$ in an effective field $B_{\perp}$ is (following Tucker 1975)

$$
T=10^{12} v^{-1 / 2} B_{\perp}^{-3 / 2} \mathrm{~s}
$$

for $v$ in $\mathrm{Hz}$ and $B_{\perp}$ in gauss. The lifetime of particles mainly responsible for radiation at $6 \mathrm{~cm}$ and at the $B$ band differ by a factor $\sim 400$. The observations require either that the radioemitting particles' streaming distance (lifetime $\times$ sin pitch angle $\times c$ ) be less than $30 \mathrm{pc}$, that the field in the detected regions is so tangled that the particles cannot move in straight lines for such distances, or that the field strength is so inhomogeneous on small scales that particles can actually escape the radiating volume before losing most of their energy. The last alternative is unlikely, due to the strong coupling (small gyroradius) of these electrons to the field structure.

The $2 \mathrm{~cm}$ data suggest that the equipartition field may be rather high, with only a few percent of the enclosed jet volume actually radiating. Even so, the weak dependence of equipartition field intensity $B($ eq) on radiating volume gives, for example, $B(\mathrm{eq})=5 \times 10^{-4}$ gauss for knot $\mathrm{D}$, with a filling factor 0.01 . This implies a lifetime for the highest energy particles seen optically $\left(v_{\text {peak }} \sim 10^{15} \mathrm{~Hz}\right)$ of slightly under $100 \mathrm{yr}$, for a streaming length of $30 \mathrm{pc}$. Thus the highest energy particles might (just) be free to move linearly along smooth field lines, but not those seen in the radio. The presence of very small-scale structure dominating the radio emission $(F . N$. Owen and P. E. Hardee, private communication) clearly suggests typical field strengths a few times higher than previously assumed, but unrealistically large fields ( $\sim 0.05$ gauss) would be required to remove the need for magnetic channeling of particles within the knots. Space Telescope observations may show whether any differential-lifetime effects are present, thus measuring characteristic scales for structure in the magnetic field.

\section{OTHER JETS: INTEGRATED PROPERTIES}

\section{a) New Measurements}

Several additional jets were detected in this study; these are described individually here, and the measurements summarized in Table 6. Notes are also given for objects not detected.

$P K S$ 0521-36.-The jet structure here is comparable to that in M87, though both larger and more luminous. Optical observations (Keel 1986; Cayatte and Sol 1987) have shown that the optical continuum is steep $(\alpha=-2$ near $5000 \AA)$ and has either a spectral break or continuous turnover. Merging the new data with those previously published shows a gradual steepening perhaps better described as a turnover than as a discrete break (Fig. 10). The listed $B$ and $V$ values are from earlier analysis (Keel 1986), as GRASP does not model adequately the strong gradients found near a very bright point source, when relatively large pixels are used. For the same reason, the new $I$ measurement pertains only to the jet outside the knot 1"6 from the core. An approximate correction could be made for the knot, based on its fractional contribution to the jet at $B, V$, and $2 \mathrm{~cm}$; the estimated total flux at $I$ is also listed in Table 6 . There is a gradient in $2 \mathrm{~cm}-0.4 \mu \mathrm{m}$ spectral index along the jet, but the optical data by themselves are not yet sufficient to show whether this represents a shift in turnover frequency as is seen in M87; this may well be, since the sense of the gradient and its amplitude are similar in both cases.

The spectroscopic data are plotted along with broad-band imaging results in Figure 10. The agreement is as close as might be expected, given the problem of scattered light from the brilliant nucleus. Observations farther to the UV will be important in specifying the full behavior of the synchrotron spectrum.

NGC 6251.- The radio jet in this galaxy is well-studied, 
TABLE 6

INTEGRATED JET FLUXES

\begin{tabular}{|c|c|c|c|c|c|}
\hline Object & Band & $\bar{\lambda}(\mu)$ & $f_{v}(\mu \mathrm{Jy})$ & Magnitude & Notes \\
\hline \multirow[t]{3}{*}{ PKS $0521-36 \ldots \ldots \ldots$} & $\boldsymbol{B}$ & 0.43 & 38 & 20.16 & From Keel 1986 \\
\hline & $V$ & 0.55 & 60 & 19.51 & From Keel 1986 \\
\hline & $I$ & 0.77 & 86 & 18.63 & Includes $40 \%$ assumed knot contribution \\
\hline \multirow{3}{*}{ 3C 66B $\ldots \ldots \ldots \ldots \ldots$} & $\boldsymbol{B}$ & 0.44 & 13.6 & 21.28 & \\
\hline & $\boldsymbol{V}$ & 0.55 & 21.5 & 20.62 & \\
\hline & $R$ & 0.67 & 36 & 19.81 & \\
\hline \multirow{5}{*}{$\begin{array}{l}\text { Coma } A \\
\text { (both knots) }\end{array}$} & $\boldsymbol{B}$ & 0.41 & 13 & 21.38 & \\
\hline & $V$ & 0.51 & $16 \pm 6$ & 20.9 & Strong emission lines \\
\hline & $R$ & 0.61 & $18^{-}$ & 20.58 & \\
\hline & $I$ & 0.83 & 26 & 19.94 & \\
\hline & $K$ & 2.03 & 143 & $\ldots$ & From P. Allan \\
\hline \multirow{3}{*}{$\begin{array}{l}\text { NGC } 6251 \ldots \ldots \ldots \ldots \\
\quad \text { (brightest knot) }\end{array}$} & $B$ & 0.45 & $1.7 \pm 0.5$ & $23.8 \pm 0.4$ & \\
\hline & $V$ & 0.56 & $3.5^{-}$ & 22.5 & \\
\hline & $R$ & 0.68 & 8.0 & 21.43 & \\
\hline $3 C 303 \ldots$. & $B$ & 0.39 & $5.5 \pm 0.5$ & 22.25 & \\
\hline \multirow{2}{*}{ (hot spot) } & $V$ & 0.48 & 6.6 & 21.91 & \\
\hline & $I$ & 0.79 & 9.1 & 21.06 & \\
\hline $3 \mathrm{C} 273$. & $B$ & 0.39 & 14.9 & 21.24 & $r=12 " .0-17 " .2$ \\
\hline \multirow[t]{3}{*}{ (inner) } & $\boldsymbol{V}$ & 0.49 & 9.5 & 21.51 & \\
\hline & $R$ & 0.59 & 16.5 & 20.66 & \\
\hline & $I$ & 0.73 & 35.9 & 19.58 & \\
\hline \multirow[t]{4}{*}{ (outer) } & $B$ & 0.39 & 11.1 & 21.43 & $r=17 " .2-21.6$ \\
\hline & $V$ & 0.49 & 13.5 & 21.12 & \\
\hline & $R$ & 0.59 & 19.0 & 20.50 & \\
\hline & $I$ & 0.73 & 44.2 & 19.35 & \\
\hline \multirow[t]{4}{*}{ (hot spot) $\ldots \ldots \ldots \ldots$} & $B$ & 0.41 & $\leqq 0.3$ : & $\geqq 25.6$ & $r=21 " .6-23 " .4$ \\
\hline & $V$ & 0.50 & 1.1 & 23.83 & \\
\hline & $R$ & 0.59 & 6.1 & 21.74 & \\
\hline & $I$ & 0.73 & 9.6 & 21.01 & \\
\hline
\end{tabular}

NoTES. $-\bar{\lambda}$ is in the objects' emitted frame.

Magnitudes are as observed, without galactic reddening correction; flux densities have been corrected. Photometric errors are taken to be in the range of $5 \%-7 \%$ unless otherwise noted.

being nearly straight on scales from 2 mas to 5 arcmin (a projected range of $1 \mathrm{pc}$ to $1 \mathrm{Mpc}$, for $\left.H_{0}=100\right)$. A weak counterjet is seen (Jones et al. 1986). The main jet is knotty, but preserves a conelike envelope of typical opening angle $3^{\circ}$ (Saunders et al. 1981; Perley, Bridle, and Willis 1984). Optical observations have shown a "light cusp" similar to that in M87, possibly due to a central mass concentration (Young et al. 1979), and evidence for a small-scale dust lane (Nieto et al. 1983). No clear detection of the jet has been reported.

The data taken here allow the recognition of excess light in $B, V$, and $R$ at the position of the strongest feature in the map shown by Perley, Bridle, and Willis (1984), as seen in Figure 2. The emission matches the radio knot in size and has optical fluxes and continuum slope comparable to those expected by analogy with other optically detected jets. The continuum shape (see Table 6 and Fig. 10) is again steeper than seen in M87, perhaps indicating a lower frequency spectral turnover.

Coma $A=3 C$ 277.3.- The possible coincidence of optical emission with a knot in the radio jet was noted by Goodson, Palimaka, and Bridle (1979). More detailed radio and optical investigations by Miley et al. (1981) and van Breugel et al. (1985) indicated that two optical emission regions are present in the jet, with both continuum and line emission; polarization implies that the continuum is primarily of synchrotron origin.

The continuum emission here is concentrated into a small area (Fig. 2); it is not completely clear whether one or two optical knots are present. These data show a slight offset between continuum and emission-line peaks, with a hint that the emission-line knots are larger.
The presence of emission lines was corrected both by the $\mathrm{H} \alpha+[\mathrm{N} \mathrm{II}]$ image and via the emission-line strengths presented by van Breugel et al. (1985). The $V$ band contribution of line emission is especially large, since at $z=0.086$ the [O III] 5007 line falls close to the center of the passband; $R$ and $I$ are relatively clean, since the $\mathrm{H} \alpha$ and [N II] lines fall in the gap between the two as defined by the Mould filters, at about $7125 \AA$. The $\lambda 7146$ image indicates that the emission lines (but not the continuum) are more extended than the apertures used for the measurements by van Breugel et al. (1985). The assumption was made that the [O III] and $\mathrm{H} \alpha$ structures are similar. On these grounds, the measured fluxes have been multiplied by $0.95,0.65,0.45$, and 1.0 (at $B, V, R$, and $I$ ) to correct for line emission; the $V$ flux is thus of relatively low weight. The remaining continuum is close to a power law, somewhat steeper than seen in the radio (van Breugel et al. 1985). Table 6 includes a $2.2 \mu \mathrm{m}$ measurement from UKIRT kindly communicated by P. Allan, taken with a $7 "$ aperture. The interpretation of the overall spectrum is complicated by the presence of two radio knots with different spectral indices, very close to the optical centroid.

3C 66B.- Radio maps from Westerbork (van Breugel 1982) and the VLA (Leahy, Jägers, and Pooley 1986) show very extended two-sided structure, with a strong (knotty) jet to the northeast. The strongest knot appears 2".5 from the core. Optical detection of several of the knots in the $B$ band was reported by Butcher, van Breugel, and Miley (1980), who suggested from the $B$-to-radio ratio that this represents synchrotron radiation. The present results are in accord with this 


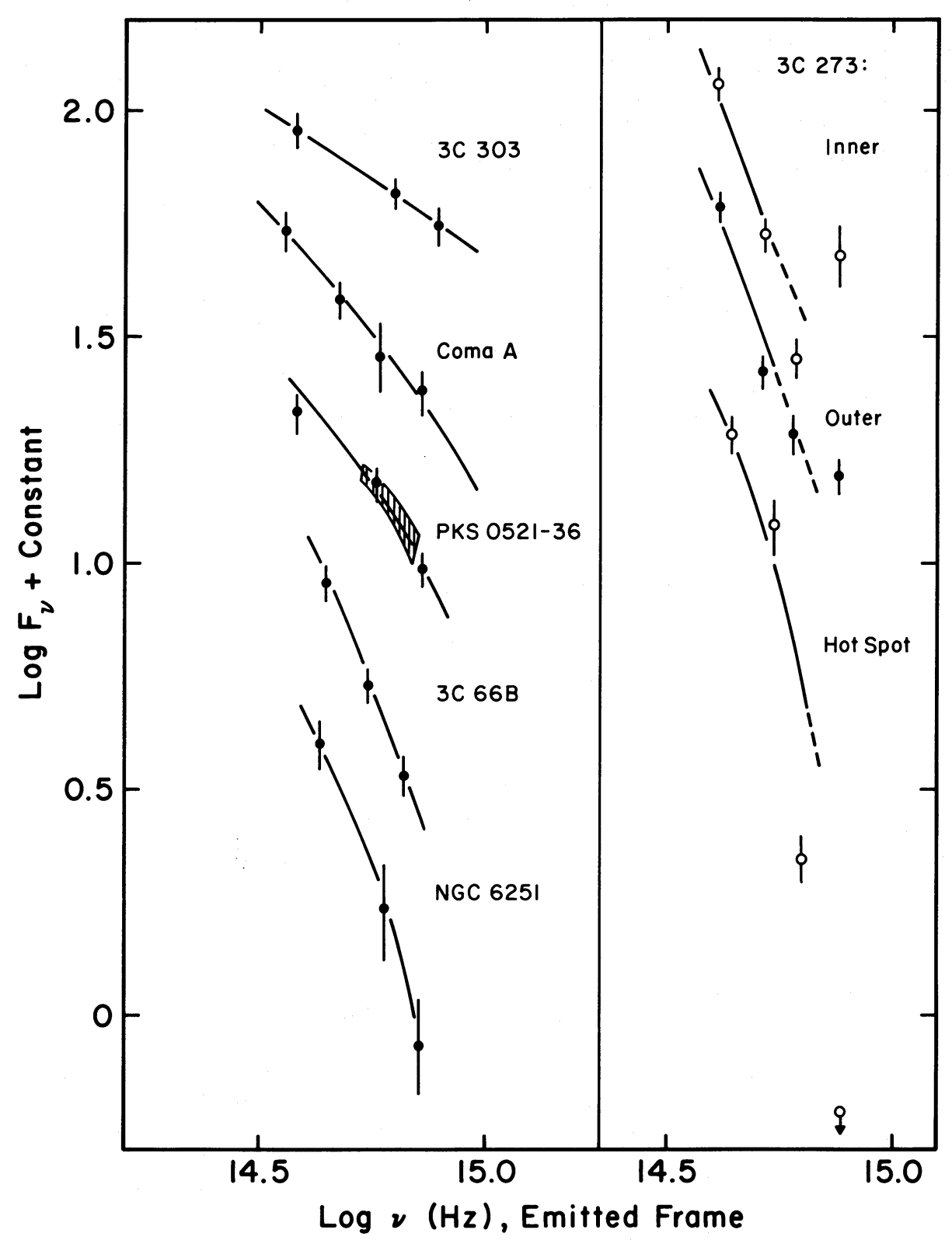

Fig. 10.-Integrated spectral energy distributions of five jets and the 3C 303 hot spot, with best-fit truncated synchrotron models (Table 7). For the steepest spectra, the fits are only approximate because finite-passband effects can become important.

interpretation, and similar to other jets; the optical continuum is steeper than the radio, and the flux level indicates that a turnover has occurred in the near-infrared.

The coarse scale of the $3 \mathrm{C} 66 \mathrm{~B}$ images here does not allow a clear detection of the innermost knot, though the jet is well seen farther out (Fig. 2). In all three bands, an additional, diffuse region is found partially projected against the jet, from 6.5 to 12 .5 $\mathrm{E}$ and $2 " .6$ to $8.5 \mathrm{~N}$ of the nucleus. Its nature is unclear; there is no corresponding structure in published radio maps. The total $V$ magnitude of this feature is about 22.0. The fluxes in Table 6 and Figure 10 refer to the whole jet from 2 " to $10^{\prime \prime}$ from the nucleus; these images do not allow a clear division into knots. The optical spectral index is $\alpha=-2.38$; the three data points are internally consistent with a single power law, but the radio flux level clearly implies an intervening turnover.
3C 303.- This distant radio galaxy was reported to show an optical object coincident with a radio hot spot by Kronberg et al. (1977), who found the optical spectrum to be devoid of strong emission features. The field contains another UV-excess object, a QSO at $z=1.57$. The optical counterpart of the hot spot itself is resolved, in the same sense as the radio structureelongation along the line toward the nucleus (Fig. 3). The optical slope of the spectrum is quite flat $(\alpha=-0.6)$, neatly continuing the radio spectrum. This is in contrast to the situation in jets proper, and more like the case in Pictor A (Röser and Meisenheimer 1987). No $R$ measurement is given, as poor seeing for this frame resulted in significant overlap of the QSO and hot spot images.

$3 C$ 273.-From multicolor photopolarimetry, Röser and Meisenheimer (1986) identify three distinct parts of the optical jet: the radio hot spot, seen only longward of $R$ with a turnover 
frequency $\sim 7 \times 10^{13} \mathrm{~Hz}$; a nearby optical knot with spectrum close to $v^{-1}$; and a "plateau" inward of this in which an unpolarized component (thus additional to the radio synchrotron continuum) must be present. Small extensions of various colors are also present.

These data also suggest a division of the optical jet into three spatial components: a very red tip (the radio hot spot) and "inner" and "outer" regions of the jet itself, divided at a local minimum in $B$ and $V 17$ ".2 from the core. The tip shows a steeply falling spectrum while both jet regions show excess light at $B$ (and perhaps $V$ ) superposed on a redder continuum (shape close to $v^{-1}$ ), as shown in Figure 10. The spectral fits shown account only for this redder continuum; the blue light is dominated by a different, unspecified mechanism.

The results here are in reasonable accord with those of Röser and Meisenheimer, and the recently published measurements by Hayes and Sadun (1987), and add to the evidence that additional optical-radiation mechanisms are active in this object which have not yet been found in other optical jets. The radio hot spot alone behaves much like jet emission in other objects, with a strong cutoff in the near-IR. The optical knot, by contrast, seems more like some other radio hot spots, with a fairly constant spectral index and evidence for X-rays from the same power law (Willingale 1981). Note, however, its lack of a matching radio peak.

PKS $0812+02$. - Optical emission has been reported from the region of this $z=0.4$ quasar's jet or lobe by Wyckoff et al. (1983). The present images indicate that the object has moderately strong redshifted [O III] 5007 emission (just at the edge of the spectral range examined by Wyckoff et al.), and shows additional emission-line structure to the south (Fig. 11 [Pl. 17]). The $B$ image indicates that the optical counterpart to the radio lobe is somewhat brighter $(B=20.67)$ than measured by Wyckoff et al., using a $3^{\prime \prime} \times 4^{\prime \prime}$ slit; it may thus be more extended than this. Its brightness in the [O III] image, coupled with the continuum shape given by Wyckoff et al., suggests an excess corresponding to a $\lambda 5007$ equivalent width (observed) of about $30 \AA$. The feature to the south may have even stronger emission, but lack of a frame in the nearby continuum makes this very uncertain.

\section{b) Nondetections}

NGC 5128.-Emission-line structure is well known in the outer jet of Cen A, $5^{\prime}-10^{\prime}$ from the nucleus (see, e.g., Graham and Price 1981). The patchy dust lane crossing the nucleus makes identification of any optical counterpart to the inner knots of the jet (Burns, Feigelson, and Schreier 1983) difficult to confirm. If the radio core is identified with the $2.2 \mu \mathrm{m}$ peak (Giles 1986), optical knots do appear that are approximately coincident with the inner radio knots, but the role of extinction variations is hard to evaluate; the apparent knots could be largely due to structures in the intervening dust. Optical spectra of these regions have been reported by Brodie, Königl, and Bowyer (1983), who found emission lines of peculiar ionization near the strongest knots. They also report a continuum excess which they interpret as a synchrotron continuum with a break, but their spectral break is too sharp for any possible synchrotron feature and is likely due to a mixed stellar population plus dust.

To avoid the uncertainties associated with structure in the dust lane, I have searched for the northern jet where it comes out from behind the dust lane. The starlight was modeled as a de Vaucouleurs profile fitted to the region outside the dust lane (taking the parameters from Dufour et al. 1979), and subtracted to seek residual emission from the jet. None was found at distances $47^{\prime \prime}-160^{\prime \prime}$ from the nucleus.

$3 C$ 31.-The radio source shows twin jets and somewhat distorted extended structure (Fomalont et al. 1980; van Breugel and Jägers 1982). Butcher, van Breugel, and Miley (1980) reported faint optical emission from the inner part of the northern jet, near the dust lane which crosses just north of the nucleus. This is not confirmed in the present data. The dust lane complicates the analysis, but its effects were localized by fixing the isophote centers in this object. Any jet emission must be a factor $\sim 4$ fainter than that in $3 \mathrm{C} 66 \mathrm{~B}$.

$3 C$ 219. - The radio map presented by Perley et al. (1980) shows a bright jet to the south, strong enough that optical emission might be seen $5^{\prime \prime}-15^{\prime \prime}$ from the core. While emission may be present in the more distant hot spots (Crane, Tyson, and Saslaw 1983), none was seen from the jet itself.

$3 C$ 296.-This source is situated in an elliptical with S0 companion. No emission was seen in the jet, as mapped by Birkinshaw, Laing, and Peacock (1981), to a level $R=24.8$ mag arcsec ${ }^{2}$, implying $\alpha<-0.98$ for pointlike knots in the jet, or $\alpha<-0.78$ if they are resolved at the published beam size.

$3 C 449$ and B2 $1321+31$. - These are symmetric twin-jet sources (Cornwell and Perley 1982; Fanti et al. 1982). No optical emission has yet been seen in any highly symmetric source; it is not yet clear whether these sources are distinct in this respect from more one-sided ones, or whether they are sufficiently rare that no suitable one has been observed. These limits do not place strong constraints on the spectral shapes, due to the low radio surface brightness: $\alpha<-0.81$ and -0.77 , respectively, for small condensations in the jet, from $B$ to 2.7 $\mathrm{GHz}$.

Pictor A.-While there is a strong optical counterpart to western hot spot (Röser and Meisenheimer 1987), no emission was found closer to the core. Parameters for the hot spot in comparisons are taken from their work.

\section{OPTICAL SYNCHROTRON TURNOVERS AND HIGH-ENERGY PARTICLES IN JETS}

As seen in the data discussed in $\S$ III for M87, and $\S$ IV for other jets that are not so well resolved, a strong steepening of the spectrum, or turnover, is seen in every jet in which we seem to be observing optical synchrotron radiation. Note that many objects could show a lower frequency turnover, in the infrared, and remain unseen so far. This type of turnover is also seen in some, but not all, hot spots with optical counterparts.

Since synchrotron spectra show only very broad-band spectral features regardless of the electron energy spectrum, direct inversion of the observed spectrum requires accurate observations over an extremely wide frequency range, only available for such objects as the Crab Nebula (Craig et al. 1985). Hence, I will present fits to simple, highly parameterized models, as an aid in comparing different objects and providing rough estimates of their physical properties.

Optically thin synchrotron spectra have been generated for electron distributions having cutoffs toward high energies of several forms, by convolving these distributions with the monoenergetic synchrotron spectrum, using the asymptotic expressions and functional tabulations given by Ginzburg and Syrovatskii (1965). Direct comparison of these spectra with the observations is more useful than trying to measure a "break amplitude," because the interpretation of a spectral break, even if such a discrete feature is present, may be obscure for realisti- 


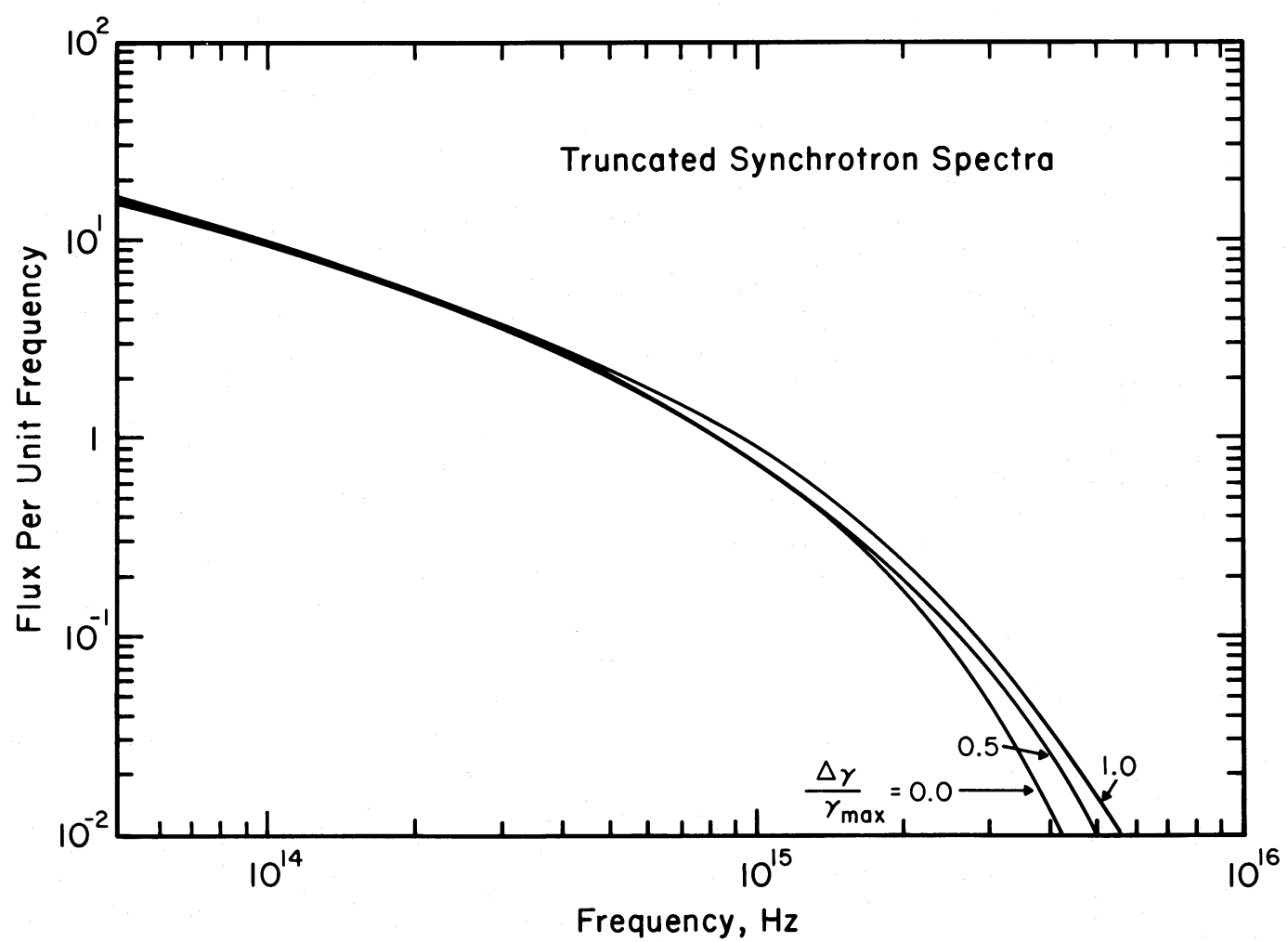

Fig. 12.- Synchrotron spectra for electron distributions with linearly ramping cutoffs of a power law, with cutoff widths relative to the highest energies of $\Delta \gamma / \gamma=0$ (sharp), 0.5 , and 1 .

cally complex sources (Pacholczyk 1975), and in any case the data do not necessarily imply broken power-law forms. It is also simpler to suppose a common spectral shape, with a single changing characteristic frequency, than a whole group of broken power laws with different amplitudes, since theoretical breaks have distinct values corresponding to various dominant modes of energy loss. This approach gives an estimate of the instantaneous electron distribution, regardless of the history of individual particles, implicitly assuming that the jets are close to a steady state in internal energy balance.

A synchrotron spectrum is impressively indifferent to the details of an energy cutoff in the radiating particles, as shown in Figure 12. A fairly gradual energy cutoff, up to about a factor 3 , is almost indistinguishable from a completely sharp one. There are in fact two free parameters in these models aside from those describing the shape of the cutoff: its effective frequency and the low-frequency spectral index. The latter is well specified from radio data for the objects in this sample and has been fixed accordingly in each case.

The best-fit spectra using a sharp energy cutoff are given in Table 7. In all cases, the critical cutoff frequency is below about $10^{15} \mathrm{~Hz}$, probably corresponding to $\gamma \sim 10^{4}$ in a typical jet.

TABLE 7

Synchrotron Models for Optically Detected Jets and Hot Spots

\begin{tabular}{|c|c|c|c|}
\hline Object & $\alpha_{10 w}$ & $v_{c}$ & Notes \\
\hline M87 D & -0.64 & $2.0 \pm 0.5 \times 10^{15}$ & \\
\hline $\mathrm{E}$ & -0.66 & $>3 \times 10^{15}$ & \\
\hline$F \ldots \ldots \ldots \ldots \ldots$ & -0.64 & $1.6 \pm 0.4 \times 10^{15}$ & \\
\hline A $\ldots \ldots \ldots \ldots \ldots$ & -0.64 & $1.2 \pm 0.1 \times 10^{15}$ & \\
\hline B $\ldots \ldots \ldots \ldots \ldots \ldots$ & -0.63 & $9.1 \pm 1.0 \times 10^{14}$ & \\
\hline $\mathrm{C} \ldots \ldots \ldots \ldots \ldots \ldots$ & -0.69 & $7.7 \pm 1.4 \times 10^{14}$ & \\
\hline PKS $0521-36 \ldots \ldots \ldots$ & -0.7 & $7.7 \pm 1.1 \times 10^{14}$ & \\
\hline $3 \mathrm{C} 66 \mathrm{~B} \ldots \ldots \ldots \ldots \ldots$ & -0.65 & $3.1 \pm 0.5 \times 10^{14}$ & \\
\hline NGC $6251 \ldots \ldots \ldots \ldots$ & -0.65 & $3.0 \pm 1.0 \times 10^{14}$ & \\
\hline Coma A .............. & -0.7 & $8.1 \pm 1.7 \times 10^{14}$ & \\
\hline 3C $303 \ldots$ & -0.60 & $>5 \times 10^{15}$ & \\
\hline Pictor A .............. & -0.60 & $>5 \times 10^{15}$ & Röser and Meisenheimer 1987 \\
\hline 3C 273 plateau ........ & -0.4 & $1.0 \times 10^{15}$ & Röser and Meisenheimer 1986 \\
\hline 3C 273 inner jet ........ & -0.6 & $2.5 \pm 0.3 \times 10^{14}$ & This work; additional blue light present \\
\hline $3 \mathrm{C} 273$ outer jet ......... & -0.6 & $2.6 \pm 0.3 \times 10^{14}$ & This work; additional blue light present \\
\hline 3C 273 hot spot ....... & -0.8 : & $2.5 \pm 0.5 \times 10^{14}$ & Röser and Meisenheimer 1986; this work \\
\hline $3 \mathrm{C} 33$ hot spot ......... & -0.8 : & $<2 \times 10^{14}$ & Meisenheimer and Röser 1986 \\
\hline
\end{tabular}

NOTES. $-\alpha_{10 w}=$ asymptotic low-frequency spectral index. $v_{c}=$ cutoff frequency in the galaxy's frame 
Values below about $2 \times 10^{14} \mathrm{~Hz}$ would result in radiation too weak for optical detection. Two hot spots, in Pictor A and 3C 303 , do show spectra without a discernable turnover in the optical range. The maximum frequency (energy) is then characteristic in particular of the radiating particles in kiloparsecscale jets. Note that the observed values may be modified by Doppler effects, if the bulk motion of the radiating matter is large. The sharpness of the observed cutoffs suggests that gradients in Doppler factor alone within the bright parts of these jets are not large.

Since the lower frequency spectra of these objects are so close to power laws, and so must the low-energy electron spectra be, one natural interpretation of the observed cutoffs is that a characteristic or limiting scale has been reached in accelerating particles to this energy, or in retaining them in the radiating region. This scale might thus be associated either with the (re)acceleration process itself, or with the structure of the magnetic field and particle distribution in the jet. In either case, the M87 jet shows that the relevant parameters change only slightly, though systematically, along the jet.

If the scale giving the cutoff is due to the jet structure, it suggests that the particles radiate (or are accelerated) only in very small or thin regions, such as the surface of the jet. As a simple example, consider particles in a slab of thickness $L$ being accelerated (and radiating) only while within the slab (zero field outside). When the gyroradius $r_{g}=m c \gamma v_{\perp} / e B$ becomes a large fraction of $L$, as it will during the lifetime of every particle reaching high energies, particle losses to the entire system will dominate the population at these energies. This process will produce a cutoff in which the distribution of energies that would otherwise be produced has a superposed cutoff in which the distribution is multiplied by a factor (1constant* $\gamma^{3 / 2}$ ), where the constant is related to $L$. The length or thickness $L$ is quite small for homogeneous conditions, only $5 \times 10^{10} \mathrm{~cm}$ in M87. If this is even roughly applicable, the emission certainly comes from regions with fine-scale structure, and perhaps from very thinly confined regions, as a thin sheath or more tangled filaments.

An alternative picture has the cutoff intrinsic to the acceleration process. Similarly, this suggests a point at which the scale-free character implied by the power-law form of the resulting energy distribution breaks down. For example, the calculations of spectra from particles accelerated in shocks performed by Heavens and Meisenheimer (1987) exhibit a sharp cutoff, mostly resulting from synchrotron-loss scales rather than specific physical processes. This cutoff is certainly sharp enough to reproduce these results for jets and was in fact first applied to the 3C 273 hot spot (Meisenheimer and Heavens 1986). Biermann and Strittmatter (1987) predict such cutoffs, in the observed frequency range, for a class of shock-acceleration models.
Note that these arguments deal only with the optically dominant population of electrons. Detection of X-rays from the jets in M87 (Schreier, Gorenstein, and Feigelson 1982) and Cen A (Feigelson et al. 1981; Burns, Feigelson, and Schreier 1983) may imply a separate population, more sparsely populated, at higher energies. The M87 X-ray measurements in fact lie above an extrapolation of the $I U E$ measurements; this in itself suggests a separate source if the X-rays in fact arise in the same region as the radio and optical synchrotron emission. The relative flux levels in the various regimes do not seem compatible with a reprocessing (Compton) origin for the $\mathrm{X}$-rays, for the local physical conditions believed to obtain in the jet (Schreier, Gorenstein, and Feigelson 1982).

\section{SUMMARY}

Optical images in several passbands have been used to study the spectral energy distributions in the continua of a number of extragalactic radio jets. In all cases, the spectrum shows a strong turnover below the extrapolated low-frequency power law. The turnovers are well fitted by a simple synchrotron model in which the electron energy distribution is sharply truncated, typically at $\gamma \sim 10^{5}$.

Image restoration has been used to examine the structure of the M87 jet at sub-arcsecond scales, and to separate various structures for spectral-index measurements. There is a systematic drop in turnover frequency going outward along the jet, broken only by unusually high values for knots $\mathrm{A}$ and $\mathrm{E}$. The optical and radio structures are everywhere the same at the 0.3 level, suggesting magnetic constraints on particle motion on scales $\sim 30$ pc.

Energy cutoffs corresponding to frequencies less than $7 \times 10^{14} \mathrm{~Hz}$ seem to be ubiquitous among radio jets, but not all observed hot spots. This may reflect a characteristic scale for the acceleration or radiating regions in jets, which may be violated by hot spots. This scale, naively estimated, is extremely small, $\sim 10^{11} \mathrm{~cm}$, and may probe very fine-scale structures in jets.

I am grateful for the support and assistance provided by $\mathbf{G}$. Will, W. Binkert, and P. Ugarte at several telescopes. Walter Jaffe, Marijn Franx, and Marco de Vos made possible the exploitation of all the necessary software. Don Hamilton, Bob Argyle, and Rudolf Le Poole obtained images on my behalf at Kitt Peak and La Palma, when I could never get clear weather in certain seasons. Frazer Owen, Rick Perley, Alan Bridle, and Guy Pooley provided radio maps of several objects for detailed comparison. I acknowledge helpful discussions with Frazer Owen, Phil Hardee, Robert Laing, and Vincent Icke. Peter Allan provided his unpublished infrared measurements of Coma A. I thank Myrna Cook, Glenn Pickens, and Joyce Du Hamel for preparing the figures in Tucson.

\section{APPENDIX A.}

\section{SURFACE PHOTOMETRY OF RADIO GALAXIES}

As a by-product of the galaxy modeling and subtraction described in $\S$ II $c$, new surface-brightness and shape profiles have been measured for most of the objects in Table 1. Several of these have no published surface photometry, so these results are presented in this Appendix.

As described earlier, the isophotes were fitted by families of ellipses, using the GRASP software essentially as described by Davis et al. (1985). The logarithmic sample spacing results in vastly different influences of the seeing profile at various parts of the galaxy profile. As an aid in evaluating this contribution, stars have been measured in as many cases as possible (only for Coma A was there 

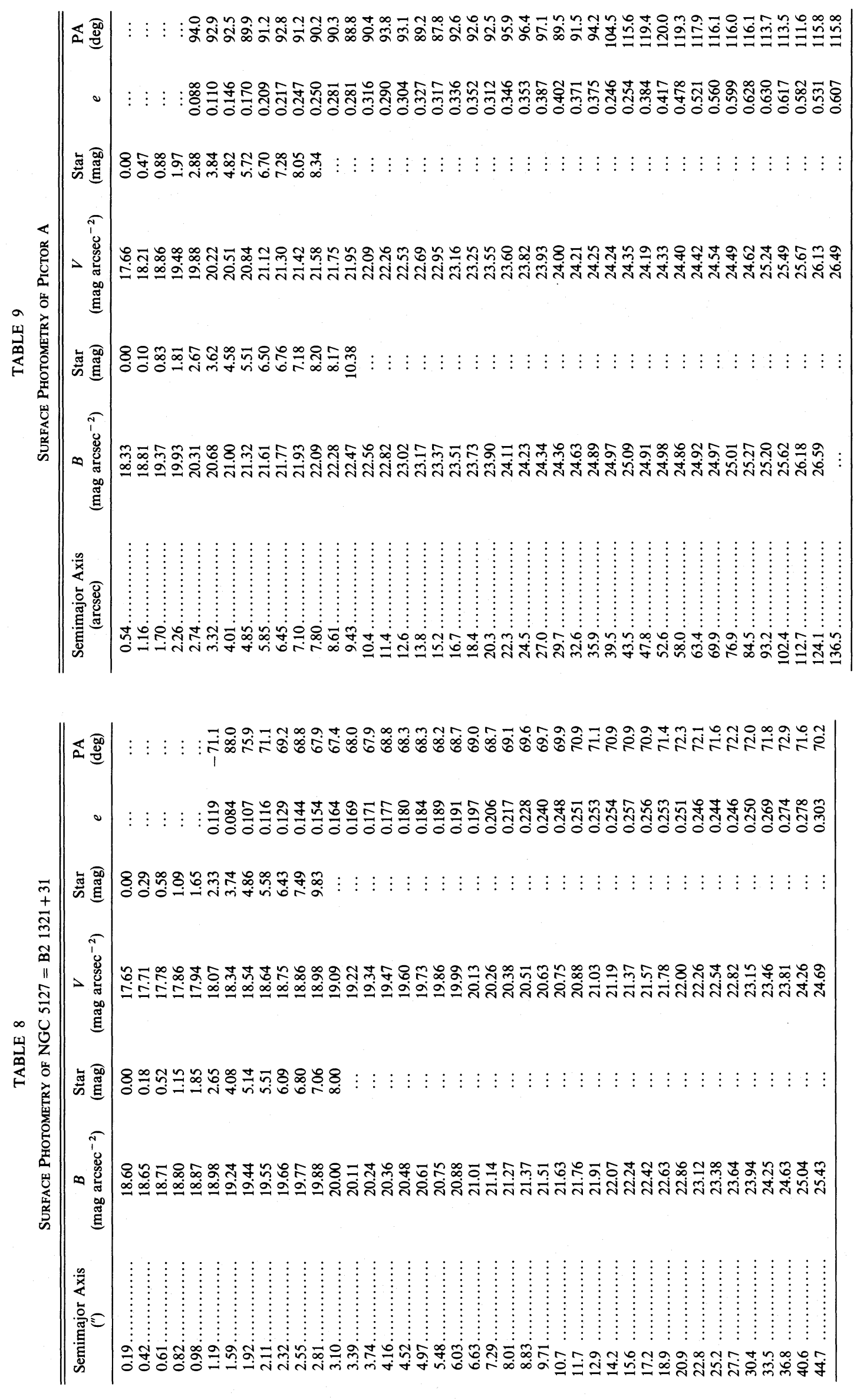

546

(C) American Astronomical Society - Provided by the NASA Astrophysics Data System 
no suitable star in the field), and the stellar surface-brightness profiles are listed next to the galaxy data under each color. Position angles are north through east, and the ellipticity is defined as 1-(axial ratio). The absolute calibration uncertainty (for the outer regions, in which seeing effects are not important) is about $5 \%$. The data are given in Tables 8-13 for six galaxies with no previous published surface photometry.

The environments of these galaxies are also of interest, in the light of recent indications that radio galaxies of high luminosity occur preferentially in interacting systems or mergers. The fields around the program galaxies contain other systems as listed below. Projected separations are computed with $H_{0}=100 \mathrm{~km} \mathrm{~s}^{-1} \mathrm{Mpc}^{-1}$.

$3 C$ 31.-There is a bright elliptical companion $33^{\prime \prime}(8 \mathrm{kpc})$ away with ellipticity 0.05 . Aside from the dust lane in $3 \mathrm{C} 31$ noted by Butcher, van Breugel, and Miley (1980), neither galaxy shows isophotal disturbances.

$3 C 66 \mathrm{~B}$. - There is a bright elliptical companion $26^{\prime \prime}(8 \mathrm{kpc})$ to the southeast. The outer isophotes of $3 \mathrm{C} 66 \mathrm{~B}$ itself are displaced to the northeast. The companion, with mean ellipticity 0.14 , shows no photometric distortions.

$3 C 219$. - This is the brightest galaxy in a cluster, with a bright elliptical projected within $5^{\prime \prime}(13 \mathrm{kpc})$ of the core. However, such an association could well be a projection effect in a cluster of this richness.

$3 C 277.3=$ Coma A.-Several fainter systems are present, starting at $17^{\prime \prime}(21 \mathrm{kpc})$ projected separation, perhaps a small group.

$3 C 296$.- S0 companion at $33^{\prime \prime}(11 \mathrm{kpc})$; this system shows a clear disk nearly edge-on.

$3 C$ 303. - Faint galaxies, not obviously related, at distances $21^{\prime \prime}(43 \mathrm{kpc})$ and greater.

$3 C$ 449. - Elliptical companion at 0.6 $(9 \mathrm{kpc})$.

NGC 6251.-Nearby disk companion NGC 6252 at $2.4(49 \mathrm{kpc})$.

$N G C 5127=B 21321+31$. - There are two very faint disk or irregular systems nearby, probably background systems.

$P K S 0521-36$. -Elliptical at $26^{\prime \prime}(21 \mathrm{kpc})$, with $V=18.48$ to the $V=26$ per square arcsecond isophote. This may be in a more extensive sparse grouping, but certainly not a cluster.

Pictor A.-No companion is seen, but the galaxy has a broad, faint tail to the northwest, associated with a drift in the outer isophotes. This is suggestive of a postmerger status.

TABLE 10

SURfaCe Photometry of 3C 303

\begin{tabular}{|c|c|c|c|c|c|c|c|c|c|c|}
\hline $\begin{array}{l}\text { Semimajor Axis } \\
\quad(\operatorname{arcsec})\end{array}$ & $\begin{array}{c}B \\
\left(\mathrm{mag}^{-\operatorname{arcsec}^{-2}}\right)\end{array}$ & Star & $\begin{array}{c}V \\
\left(\operatorname{mag}_{\operatorname{arcsec}^{-2}}\right)\end{array}$ & Star & $\begin{array}{c}R \\
\left(\mathrm{mag}_{\operatorname{arcsec}^{-2}}\right)\end{array}$ & Star & $\begin{array}{c}I \\
\left(\mathrm{mag} \mathrm{arcsec}^{-2}\right)\end{array}$ & Star & $e$ & $\begin{array}{l}\text { PA } \\
\text { (deg) }\end{array}$ \\
\hline $0.19 \ldots$ & 19.89 & 0.00 & 19.08 & 0.00 & $\ldots$ & $\ldots$ & $\ldots$ & $\ldots$ & $\ldots$ & $\ldots$ \\
\hline $0.42 \ldots \ldots \ldots \ldots \ldots$ & 20.06 & 0.01 & 19.26 & & & & & & $\ldots$ & $\ldots$ \\
\hline $0.61 \ldots \ldots \ldots \ldots$ & 20.34 & 0.35 & 19.53 & 0.35 & 19.01 & 0.35 & 18.10 & 0.35 & $\ldots$ & $\ldots$ \\
\hline $0.82 \ldots \ldots \ldots \ldots$ & 20.76 & 0.92 & 19.79 & $\ldots$ & 19.31 & 0.69 & 18.37 & 0.76 & $\ldots$ & $\ldots$ \\
\hline $0.98 \ldots$ & 21.08 & 1.41 & 20.08 & 1.60 & 19.62 & 0.99 & 18.65 & 1.22 & $\ldots$ & $\ldots$ \\
\hline $1.19 \ldots$ & 21.43 & 1.90 & 20.40 & 2.17 & 19.94 & 1.32 & 18.99 & 1.76 & & \\
\hline $1.59 \ldots \ldots \ldots \ldots$ & 22.02 & 2.93 & 21.01 & 3.30 & 20.49 & 1.86 & 19.59 & 2.95 & 0.125 & 5.2 \\
\hline $1.92 \ldots \ldots \ldots \ldots$ & 22.48 & 4.09 & 21.44 & 4.32 & 20.92 & 2.24 & 20.04 & 3.88 & 0.108 & 13.8 \\
\hline $2.11 \ldots \ldots$ & 22.70 & 4.61 & 21.65 & 4.80 & 21.01 & 2.42 & 20.27 & 4.35 & 0.100 & 15.3 \\
\hline $2.32 \ldots \ldots \ldots \ldots \ldots$ & 22.94 & 4.98 & 21.86 & 5.38 & 21.23 & 2.62 & 20.46 & 4.61 & 0.085 & 19.3 \\
\hline $2.55 \ldots \ldots \ldots \ldots$ & 23.10 & 5.36 & 22.07 & 6.18 & 21.43 & 2.80 & 20.64 & 4.76 & 0.091 & 22.3 \\
\hline $2.81 \ldots \ldots \ldots \ldots$ & 23.33 & 5.77 & 22.27 & 6.33 & 21.61 & 2.98 & 20.88 & 5.01 & 0.092 & 27.3 \\
\hline $3.10 \ldots \ldots \ldots \ldots$ & 23.47 & 6.24 & 22.43 & 7.09 & 21.82 & 3.19 & 21.05 & 5.24 & 0.122 & 22.0 \\
\hline $3.39 \ldots \ldots \ldots \ldots \ldots$ & 23.69 & 6.47 & 22.60 & 7.29 & 22.01 & 3.39 & 21.24 & $\ldots$ & 0.097 & 23.6 \\
\hline $3.74 \ldots \ldots \ldots \ldots \ldots$ & 23.90 & $\ldots$ & 22.81 & 7.28 & 22.18 & 3.55 & 21.46 & $\ldots$ & 0.087 & 27.6 \\
\hline $4.11 \ldots \ldots \ldots \ldots$ & 24.03 & $\ldots$ & 22.99 & 8.54 & 22.35 & 3.73 & 21.63 & $\ldots$ & 0.126 & 21.3 \\
\hline $4.52 \ldots \ldots \ldots \ldots \ldots$ & 24.21 & $\begin{array}{l}\cdots \\
\cdots\end{array}$ & 23.20 & $\ldots$ & 22.52 & 3.89 & 21.86 & $\ldots$ & 0.123 & 16.3 \\
\hline $4.97 \ldots \ldots \ldots \ldots \ldots$ & 24.31 & $\ldots$ & 23.40 & $\ldots$ & 22.68 & 4.06 & 22.08 & $\ldots$ & 0.153 & 12.7 \\
\hline $5.48 \ldots \ldots \ldots \ldots$ & 24.55 & $\ldots$ & 23.57 & $\ldots$ & 22.88 & 4.26 & 22.28 & $\ldots$ & 0.146 & 1.9 \\
\hline $5.85 \ldots \ldots \ldots \ldots$ & 24.79 & $\ldots$ & 23.85 & $\ldots$ & 23.09 & 4.46 & 22.48 & $\ldots$ & 0.158 & -2.0 \\
\hline $6.63 \ldots \ldots \ldots \ldots$ & 24.97 & $\begin{array}{l}\ldots \\
\ldots\end{array}$ & 24.11 & $\ldots$ & 23.25 & 4.59 & 22.73 & $\ldots$ & 0.168 & -7.3 \\
\hline $7.29 \ldots \ldots \ldots \ldots \ldots$ & 25.15 & $\ldots$ & 24.30 & $\ldots$ & 23.43 & 4.80 & 23.14 & $\ldots$ & 0.200 & -5.9 \\
\hline $8.01 \ldots \ldots \ldots \ldots$ & 25.21 & $\ldots$ & 24.49 & $\ldots$ & 23.64 & 5.01 & 23.42 & $\ldots$ & 0.249 & 2.2 \\
\hline $8.33 \ldots \ldots \ldots \ldots$ & 25.43 & $\ldots$ & 24.73 & $\ldots$ & 23.80 & $\ldots$ & 23.65 & $\ldots$ & 0.273 & 1.5 \\
\hline $9.71 \ldots \ldots \ldots \ldots$ & 25.81 & $\ldots$ & 25.05 & $\ldots$ & 24.08 & $\ldots$ & 23.88 & $\ldots$ & 0.234 & 6.0 \\
\hline $10.7 \ldots \ldots \ldots \ldots \ldots$ & 25.98 & $\ldots$ & 25.31 & $\ldots$ & 24.27 & $\ldots$ & 24.18 & $\ldots$ & 0.244 & 7.6 \\
\hline $11.7 \ldots \ldots \ldots \ldots \ldots$ & 26.13 & $\ldots$ & 25.65 & $\ldots$ & 24.31 & $\ldots$ & 24.46 & $\ldots$ & 0.237 & 5.9 \\
\hline $12.9 \ldots \ldots \ldots \ldots$ & 26.44 & $\ldots$ & 26.01 & $\ldots$ & 24.43 & $\ldots$ & 24.96 & $\ldots$ & 0.220 & 8.2 \\
\hline $14.2 \ldots \ldots \ldots \ldots \ldots$ & 26.36 & $\ldots$ & 26.03 & $\ldots$ & 24.63 & $\ldots$ & 25.30 & $\ldots$ & 0.270 & 10.7 \\
\hline $15.6 \ldots \ldots \ldots \ldots \ldots$ & 26.52 & $\ldots$ & 26.40 & $\ldots$ & 24.74 & $\ldots$ & 25.68 & $\ldots$ & 0.269 & 11.2 \\
\hline $17.2 \ldots \ldots \ldots \ldots \ldots$ & 26.77 & $\ldots$ & 27.01 & $\ldots$ & 24.78 & $\ldots$ & $\ldots$ & $\ldots$ & 0.261 & 7.1 \\
\hline $18.9 \ldots \ldots \ldots \ldots$ & 26.74 & $\ldots$ & $\ldots$ & $\ldots$ & 24.86 & $\ldots$ & $\ldots$ & $\ldots$ & 0.274 & 10.8 \\
\hline
\end{tabular}



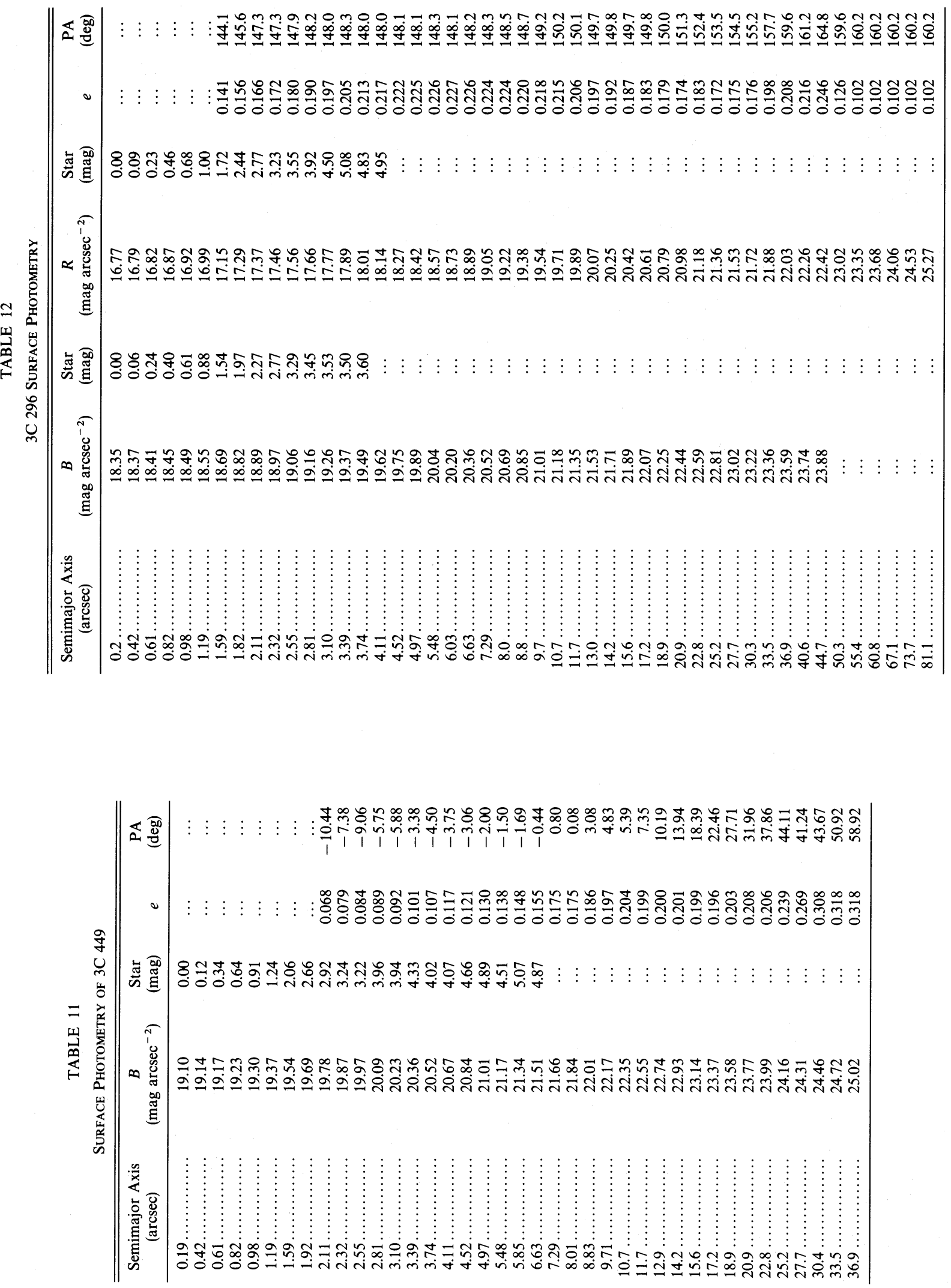
TABLE 13

SURfaCe Photometry of 3C 66B

\begin{tabular}{|c|c|c|c|c|c|c|c|}
\hline $\begin{array}{l}\text { Semimajor Axis } \\
\text { (arcsec) }\end{array}$ & $\begin{array}{c}B \\
\left(\mathrm{mag}_{\operatorname{arcsec}^{-2}}\right)\end{array}$ & $\begin{array}{c}\text { Star } \\
(\mathrm{mag})\end{array}$ & $\begin{array}{c}V \\
\left(\mathrm{mag} \operatorname{arcsec}^{-2}\right)\end{array}$ & $\begin{array}{c}R \\
\left(\mathrm{mag}_{\left.\operatorname{arcsec}^{-2}\right)}\right.\end{array}$ & $\begin{array}{c}\text { Star } \\
(\mathrm{mag})\end{array}$ & $e$ & $\begin{array}{c}\text { PA } \\
(\mathrm{deg})\end{array}$ \\
\hline 0.39 . & $\ldots$ & $\ldots$ & 18.45 & 17.70 & 0.00 & $\ldots$ & $\ldots$ \\
\hline $0.69 \ldots \ldots \ldots \ldots$ & $\cdots$ & $\cdots$ & 18.52 & 17.77 & 0.31 & $\ldots$ & $\cdots$ \\
\hline $1.01 \ldots \ldots \ldots \ldots$ & $\ldots$ & $\ldots$ & 18.62 & 17.89 & 0.73 & $\ldots$ & $\ldots$ \\
\hline $1.48 \ldots \ldots \ldots \ldots \ldots$ & $\cdots$ & $\cdots$ & 18.81 & 18.10 & 1.55 & $\ldots$ & $\cdots$ \\
\hline $1.79 \ldots \ldots \ldots \ldots \ldots$ & $\cdots$ & $\cdots$ & 18.95 & 18.24 & 2.16 & $\ldots$ & $\cdots$ \\
\hline $2.17 \ldots \ldots \ldots \ldots \ldots$ & 20.30 & 2.62 & 19.12 & 18.42 & 2.91 & 0.052 & $-\ddot{83.7}$ \\
\hline $2.39 \ldots \ldots \ldots \ldots$ & 20.35 & 3.12 & 19.21 & 18.52 & 3.33 & 0.051 & -77.7 \\
\hline $2.62 \ldots \ldots \ldots \ldots \ldots$ & 20.41 & 3.72 & 19.32 & 18.63 & 3.75 & 0.060 & -82.9 \\
\hline $2.89 \ldots \ldots \ldots \ldots$ & 20.49 & 4.18 & 19.43 & 18.74 & 4.20 & 0.071 & -85.3 \\
\hline $3.17 \ldots \ldots \ldots \ldots$ & 20.59 & 4.61 & 19.54 & 18.85 & 4.61 & 0.078 & -86.7 \\
\hline $3.49 \ldots \ldots \ldots \ldots$ & 20.70 & 5.02 & 19.67 & 18.97 & 5.07 & 0.080 & -88.4 \\
\hline $3.84 \ldots \ldots \ldots \ldots$ & 20.83 & 5.51 & 19.80 & 19.09 & 5.48 & 0.074 & 88.6 \\
\hline $4.21 \ldots \ldots \ldots \ldots \ldots$ & 20.96 & 6.10 & 19.92 & 19.22 & 5.89 & 0.080 & 85.9 \\
\hline $4.64 \ldots \ldots \ldots \ldots \ldots$ & 21.08 & 6.23 & 20.05 & 19.36 & 6.19 & 0.071 & 82.2 \\
\hline $5.11 \ldots \ldots \ldots \ldots \ldots$ & 21.21 & $\ldots$ & 20.18 & 19.48 & 6.64 & 0.071 & 78.0 \\
\hline $5.62 \ldots \ldots \ldots \ldots$ & 21.34 & $\ldots$ & 20.31 & 19.62 & 6.82 & 0.065 & 70.3 \\
\hline $6.20 \ldots \ldots \ldots \ldots \ldots$ & 21.45 & $\ldots$ & 20.44 & 19.76 & 7.09 & 0.059 & 62.7 \\
\hline $6.79 \ldots \ldots \ldots \ldots$ & 21.59 & $\ldots$ & 20.58 & 19.89 & 7.59 & 0.054 & 56.9 \\
\hline $7.49 \ldots \ldots \ldots \ldots$ & 21.74 & $\cdots$ & 20.71 & 20.02 & 7.89 & 0.050 & 44.2 \\
\hline $8.23 \ldots \ldots \ldots \ldots$ & 21.86 & $\ldots$ & 20.85 & 20.15 & $\ldots$ & 0.052 & 35.7 \\
\hline $9.05 \ldots \ldots \ldots \ldots$ & 21.99 & $\cdots$ & 20.98 & 20.28 & $\ldots$ & 0.070 & 28.3 \\
\hline $9.95 \ldots \ldots \ldots \ldots \ldots$ & 22.12 & $\ldots$ & 21.12 & 20.42 & $\ldots$ & 0.095 & 26.6 \\
\hline $10.96 \ldots \ldots \ldots \ldots \ldots$ & 22.23 & $\ldots$ & 21.24 & 20.55 & $\ldots$ & 0.097 & 27.0 \\
\hline $12.05 \ldots \ldots \ldots \ldots \ldots$ & 22.37 & $\cdots$ & 21.38 & 20.68 & $\cdots$ & 0.089 & 31.5 \\
\hline $13.26 \ldots \ldots \ldots \ldots \ldots$ & 22.48 & $\ldots$ & 21.49 & 20.80 & $\ldots$ & 0.105 & 33.1 \\
\hline $14.6 \ldots \ldots \ldots \ldots \ldots$ & 22.60 & $\ldots$ & 21.63 & 20.96 & $\ldots$ & 0.118 & 35.4 \\
\hline $16.0 \ldots \ldots \ldots \ldots \ldots$ & 22.72 & $\cdots$ & 21.77 & 21.13 & $\cdots$ & 0.110 & 37.8 \\
\hline $17.7 \ldots \ldots \ldots \ldots \ldots$ & 22.83 & $\cdots$ & 21.92 & 21.19 & $\ldots$ & 0.126 & 38.1 \\
\hline $19.4 \ldots \ldots \ldots \ldots \ldots$ & 22.95 & $\cdots$ & 22.07 & 21.35 & $\cdots$ & 0.135 & 40.3 \\
\hline $21.4 \ldots \ldots \ldots \ldots \ldots$ & 23.08 & $\ldots$ & 22.24 & 21.55 & $\ldots$ & 0.141 & 39.9 \\
\hline $23.5 \ldots \ldots \ldots \ldots \ldots$ & 23.21 & $\cdots$ & 22.39 & 21.75 & $\ldots$ & 0.134 & 39.7 \\
\hline $25.9 \ldots \ldots \ldots \ldots \ldots$ & 23.33 & $\ldots$ & 22.53 & 21.90 & $\ldots$ & 0.146 & 38.3 \\
\hline $28.4 \ldots \ldots \ldots \ldots \ldots$ & 23.46 & $\ldots$ & 22.65 & 22.02 & $\ldots$ & 0.157 & 38.0 \\
\hline $31.3 \ldots \ldots \ldots \ldots \ldots$ & 23.57 & $\ldots$ & 22.74 & 22.14 & $\ldots$ & 0.178 & 37.1 \\
\hline $34.4 \ldots \ldots \ldots \ldots \ldots$ & 23.68 & $\cdots$ & 22.77 & 22.28 & $\cdots$ & 0.198 & 35.4 \\
\hline $37.8 \ldots \ldots \ldots \ldots \ldots$ & 23.75 & $\ldots$ & 22.95 & 22.41 & $\ldots$ & 0.208 & 33.1 \\
\hline $41.7 \ldots \ldots \ldots \ldots \ldots$ & 23.85 & $\ldots$ & 23.12 & 22.58 & $\ldots$ & 0.221 & 37.6 \\
\hline $45.6 \ldots \ldots \ldots \ldots \ldots$ & 23.94 & $\ldots$ & 23.31 & 22.75 & $\ldots$ & 0.234 & 35.4 \\
\hline $50.3 \ldots \ldots \ldots \ldots \ldots$ & 24.05 & $\ldots$ & 23.49 & 22.95 & $\ldots$ & 0.227 & 36.4 \\
\hline $55.4 \ldots \ldots \ldots \ldots \ldots$ & 24.16 & $\ldots$ & 23.64 & 23.07 & $\ldots$ & 0.244 & 34.4 \\
\hline $60.8 \ldots \ldots \ldots \ldots \ldots$ & 24.27 & $\ldots$ & 23.80 & 23.47 & $\ldots$ & 0.238 & 37.3 \\
\hline $67.1 \ldots \ldots \ldots \ldots \ldots$ & 24.39 & $\ldots$ & 24.10 & 24.01 & $\ldots$ & 0.227 & 40.2 \\
\hline $73.7 \ldots \ldots \ldots \ldots$ & 24.47 & $\cdots$ & 24.27 & $\ldots$ & $\ldots$ & 0.243 & 37.6 \\
\hline $81.1 \ldots \ldots \ldots \ldots \ldots$ & 24.56 & $\ldots$ & 24.10 & $\ldots$ & $\ldots$ & 0.253 & 42.1 \\
\hline $89.3 \ldots \ldots \ldots \ldots \ldots$ & 24.62 & $\ldots$ & 24.64 & $\ldots$ & $\ldots$ & 0.273 & 39.1 \\
\hline $98.3 \ldots \ldots \ldots \ldots \ldots$ & 24.67 & $\ldots$ & 24.28 & $\ldots$ & $\ldots$ & 0.273 & 39.1 \\
\hline
\end{tabular}

\section{REFERENCES}

Arp, H. C., and Lorre, J. 1976, Ap. J., 210, 58.

Baade, W. 1956, Ap. J., 123, 550

Baade, W., and Minkowski, R. 1954, Ap. J., 119, 221.

Bender, R., and Möllenhoff, C. 1987, Astr. Ap., 177, 71

Biermann, P., and Strittmatter, P. A. 1987, Ap. J., 322, 643.

Biretta, J. A., Owen, F. N., and Hardee, P. E. 1983, Ap. J. (Letters), 274, L27.

Birkinshaw, M., Laing, R. A., and Peacock, J. A. 1981, M.N.R.A.S., 197, 253

Bridle, A. H., and Perley, R. A. 1984, Ann. Rev. Astr. Ap. 22, 319.

Brodie, J., Königl, A., and Bowyer, S. 1983, Ap. J., 273, 154.

Burns, J. O., Feigelson, E. D., and Schreier, E. J. 1983, Ap. J., 273, 128.

Burstein, D., and Heiles, C. 1984, Ap. J. Suppl., 54, 33.

Butcher, H. R., van Breugel, W. J. M., and Miley, G. 1980, Ap. J., 235, 749.

Cayatte, V., and Sol, H. 1987, Astr. Ap., 171, 25.

Christian, C. A., Adams, M., Barnes, J. V., Butcher, H., Hayes, D. S., Mould, J. R., and Siegel, M. 1985, Pub. A.S.P., 97, 363.

Clark, B. G. 1980, Astr. Ap., 83, 377.

Cornwell, T. J., and Perley, R. A. 1982, in IAU Symposium 97 Extragalactic Radio Sources, ed. D. S. Heeschen and C. M. Wade, (Dordrecht: Reidel), p. 139.

Craig, I. J. D., McClements, K. G., Thompson, A. M., and Brown, J. C. 1985, Astr. Ap., 149, 171.

Crane, P., Tyson, J. A., and Saslaw, W. C. 1983, Ap. J., 265, 681.
Danziger, I. J., Fosbury, R. A. E., Goss, W. M., and Ekers, R. D. 1979, M.N.R.A.S., 188, 415

Davis, L. E., Cawson, M., Davies, R. L., and Illingworth, G. 1985, A.J. 90, 169. de Vaucouleurs, G., Angione, R., and Fraser, C. W. 1968, Ap. Letters, 2, 141. de Vaucouleurs, G., and Nieto, J.-L. 1979, Ap. J., 231, 364.

D'Odorico, S., and Dekker, H. 1986, in Optimization of the Use of CCD Detectors in Astronomy, ed. J. P. Baluteau and S. d'Odorico (Garching bei München: ESO), p. 315.

Dufour, R. J., van den Bergh, S., Harvel, C. A., Martins, D. H., Schiffer, F. H., III, Talbot, R. J., Jr., Talent, D. L., and Wells, D. C. 1979, A.J., 84, 284.

Fanti, R., Lori, C., Parma, P., Bridle, A. H., Ekers, R. D., and Fomalont, E. B. 1982, Astr. Ap., 110, 169

Feigelson, E. D., Schreier, E. J., Delvaille, J. P., Giacconi, R., Grindlay, J. G., and Lightman, A. P. 1981, Ap. J., 252, 31 .

Filippenko, A. V. 1982, Pub. A.S.P., 94, 715.

Fomalont, E. B., Bridle, A. H., Willis, A. G., and Perley, R. A. 1980, Ap. J., 237, 418.

Giles, A. B. 1986, M.N.R.A.S., 218, 165

Ginzburg, V. L., and Syrovatskii, S. I. 1965, Ann. Rev. Astr. Ap., 3, 297.

Goodson, R. E., Palimaka, J. J., and Bridle, A. H. 1979, Astr. Ap., 84, 1111.

Graham, J. A., and Price, R. M. 1981, Ap. J., 247, 813.

Hayes, J. J. E., and Sadun, A. C. 1987, A.J., 94, 871 . 
Heavens, A. F., and Meisenheimer, K. 1987, M.N.R.A.S. 225, 335

Huchra, J. P., and Brodie, J. 1984, Ap. J., 280, 547.

Johnson, H. 1966, Ann. Rev. Astr. Ap., 4, 193.

Jones, D. L., et al. 1986, Ap. J., 305, 684 .

Keel, W. C. 1984, Ap. J., 279, 550 1986, Ap.J., 302, 296

Kinman, T. D., Grasdalen, G., and Rieke, G. H. 1974, Ap. J.(Letters), 194, L1. Kronberg, P. P. 1976, Ap. J.(Letters), 203, L47.

Kronberg, P. P., Burbidge, E. M., Smith, H. E., and Strom, R. G. 1977, Ap. J., 218, 8

Leahy, J. P., Jägers, W. J., and Pooley, G. G. 1986, Astr. Ap., 156, 234

Longo, G., and de Vaucouleurs, A. 1983, A General Catalogue of Photoelectric Magnitudes and Colors in the $U, B, V$ System, (Univ. Texas Monographs Astr., 3).

Lorre, J., and Nieto, J.-L. 1984, Astr. Ap. 130, 167

Meisenheimer, K., and Heavens, A. F. 1986, Nature, 323, 419.

Meisenheimer, K., and Röser, H.-J. 1986, Nature, 319, 459.

Miley, G. K., Heckman, T. M., Butcher, H. R., and van Breugel, W. J. M. 1981, Ap. J. (Letters), 247, L5.

Narayan, R., and Nityananda, R. 1986, Ann. Rev. Astr. Ap., 24, 127.

Nieto, J.-L., Coupinot, G., Lelievre, G., and Madsen, C. 1983, M.N.R.A.S., 203, 39P.

Nieto, J.-L., and Lelievre, G. 1982, Astr. Ap., 109, 95

Pacholczyk, A. G. 1970, Radio Astrophysics (San Francisco: Freeman), p. 143

Perley, R. A., Bridle, A. H., and Willis, A. G. 1984, Ap. J. Suppl., 54, 291. Perley, R. A., Bridle, A. H., Willis, A. G., and Fomalont, E. B. 1980, Astr. J., 85,

Perola, G. C., and Tarenghi, M. 1980, Ap. J., 240, 447.
Pronik, V. I., Pronik, I. I., and Chuvaev, K. K. 1968, Soviet Astr., 11, 777.

Pronik, V., and Shcherbalov, A. 1972, Astrofizika, 8, 337.

Röser, H.-J., and Meisenheimer, K. 1986, Astr. Ap., 154, 15. 1987, Ap. J., 314, 70

Saslaw, W. C., Tyson, J. A., and Crane, P. 1978, Ap. J., 222, 433.

Saunders, R., Baldwin, J. E., Pooley, G. G., and Warner, P. J. 1981, M.N.R.A.S., 197, 287.

Schmidt, G. D., Peterson, B. M., and Beaver, E. A. 1978, Ap. J. (Letters), 220 , L31.

Schreier, E. J., Gorenstein, P., and Feigelson, E. D. 1982, Ap. J., 262, 42.

Shklovskii, I. S. 1984, Astr. Zh., 61, 833 .

Simkin, S. M. 1978, Ap. J. (Letters), 222, L55.

Stocke, J. T., Rieke, G. H., and Lebofsky, M. J. 1981, Nature, 294, 319.

Sulentic, J. W., Arp, H. C., and Lorre, J. 1979, Ap. J., 233, 44.

Tucker, W. H. 1975, Radiation Processes in Astrophysics (Cambridge: MIT Press), p. 133.

van Breugel, W. J. M. 1982, Astr. Ap., 110, 225.

van Breugel, W. J. M., and Jägers, W. 1982, Astr. Ap. Suppl., 49, 529.

van Breugel, W. J. M., Miley, G., Heckman, T., Butcher, H., and Bridle, A 1985, Ap. J., 290, 496.

Warren-Smith, R. F., King, D. J., and Scarrott, S. M. 1984, M.N.R.A.S., 210 , 415.

Willingale, R. 1981, M.N.R.A.S., 194, 359

Wyckoff, S., Johnston, K., Ghigo, F., Rudnick, L., Wehinger, P., and Boksenberg, A. 1983, Ap. J., 265, 43. Young, P. J., Sargent, W. L. W., Kristian, J., and Westphal, J. E. 1979, Ap. J.,
234, 76.

William C. KeEL: Department of Physics and Astronomy, University of Alabama, P.O. Box 1921, Tuscaloosa, AL 35487 
PLATE 13

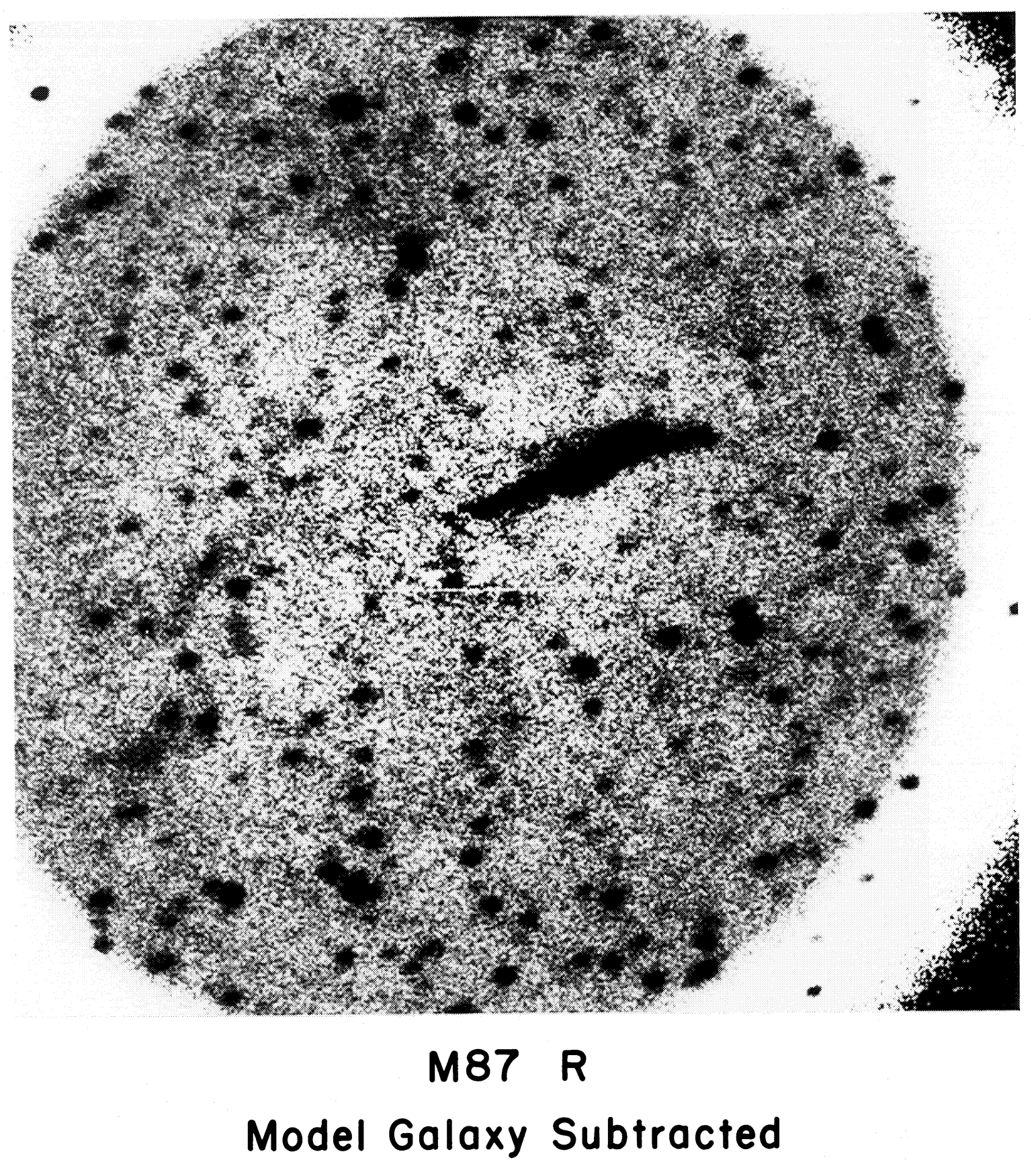

FIG. 1.-High-contrast display of an M87 image in $R$, after subtraction of a model galaxy. The subtraction has been very successful, as may be judged from the lack of systematic residuals around the jet; numerous globular clusters and $\mathrm{H} \alpha$ filaments are visible. The ring at the edge of the image reflects the limit in radius covered by the model. An area $100^{\prime \prime}$ square is shown.

KEEL (see 329, 535) 

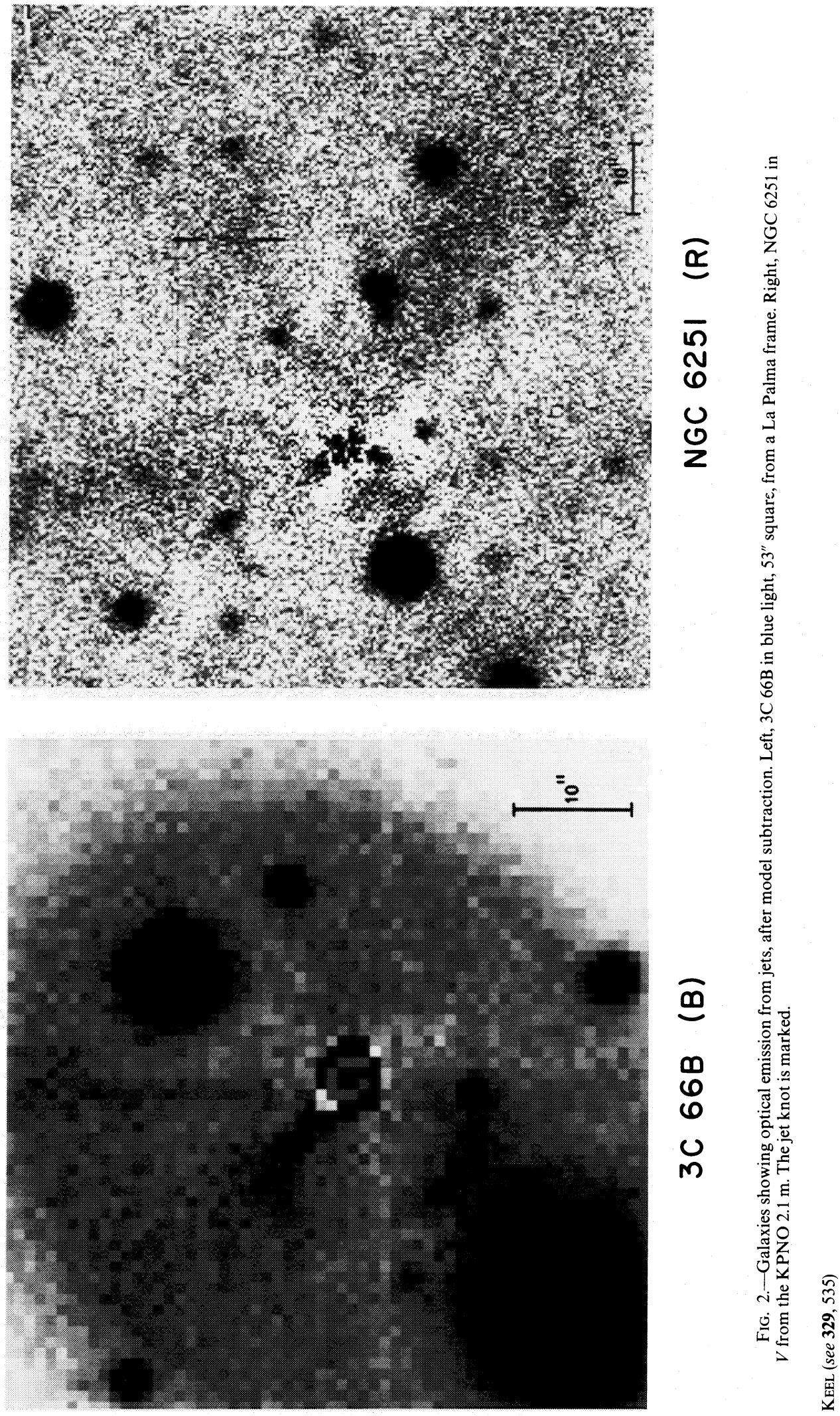
PLATE 15

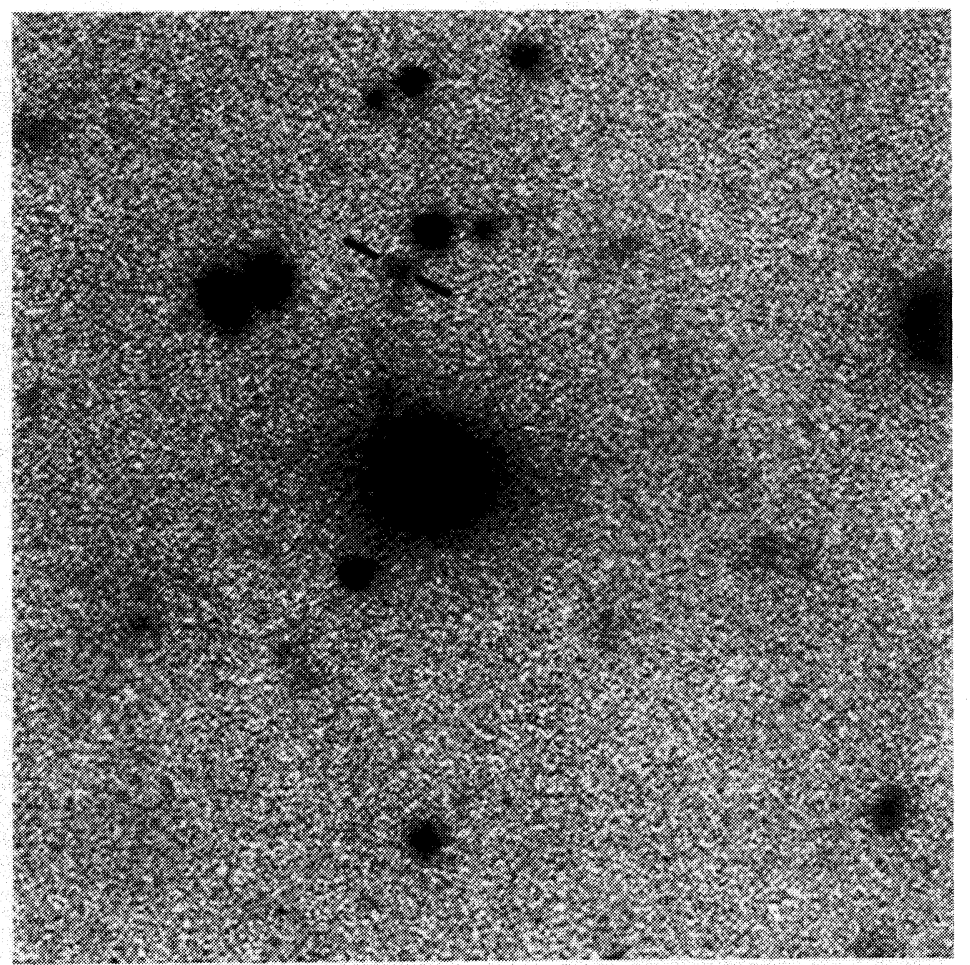

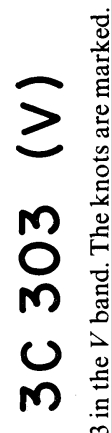
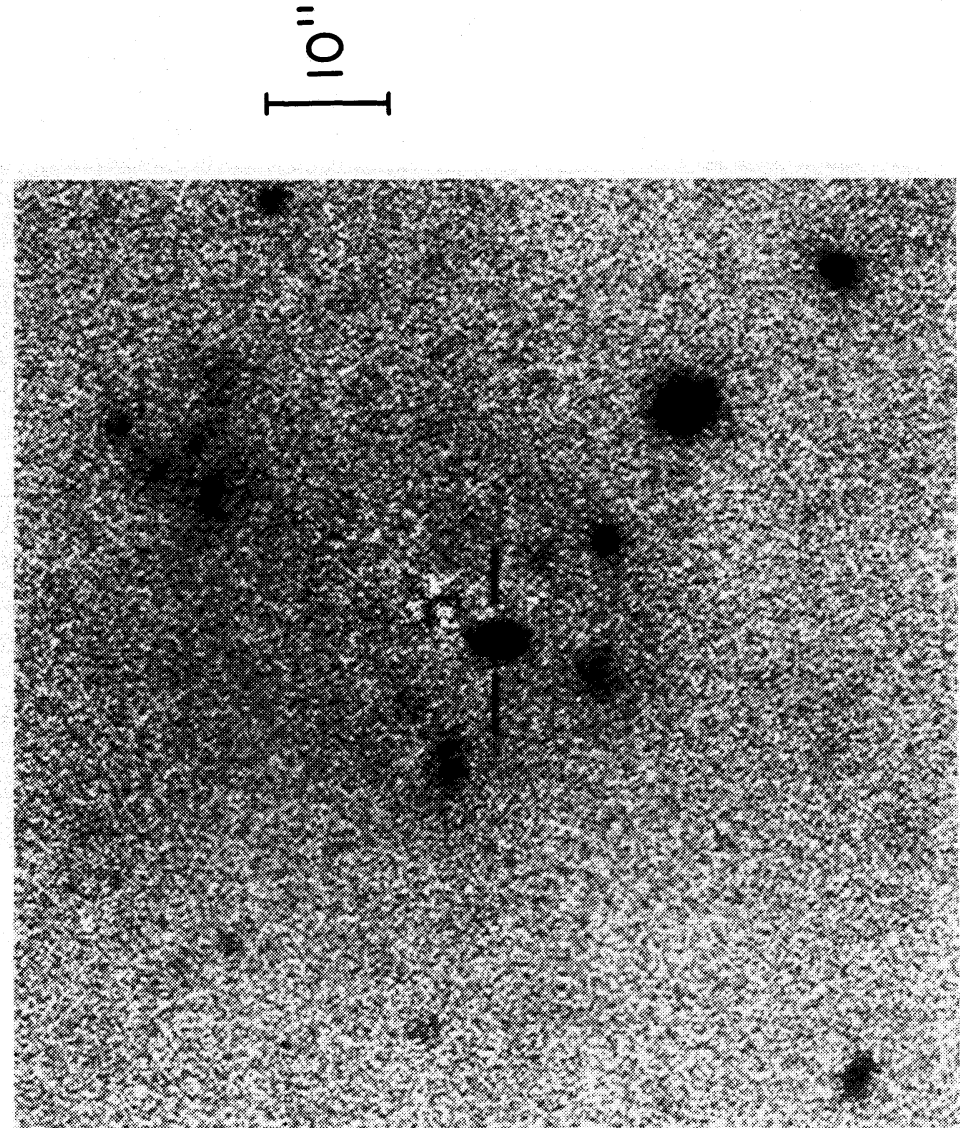

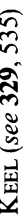




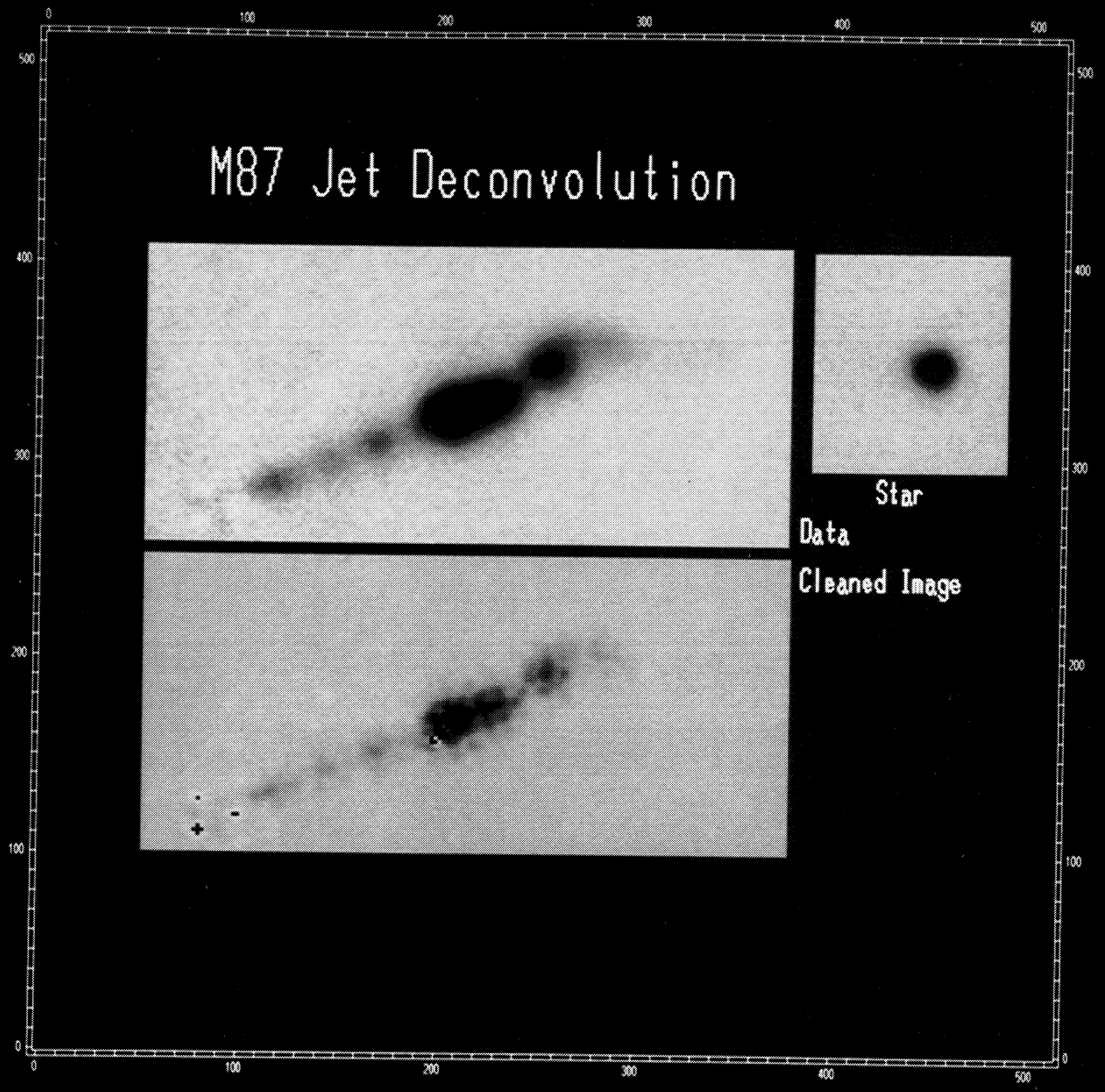

FIG. 4.- Results of CLEAN deconvolution of the M87 jet, with the $B$ data shown as an example. Top, the galaxy-subtracted jet image and a reference star image. Bottom, the CLEANed model, convolved with a 0"4 FWHM restoring profile. Very fine-scale structures here are not reliable, but overall knot shapes and fluxes are.

Keel (see 329, 536) 


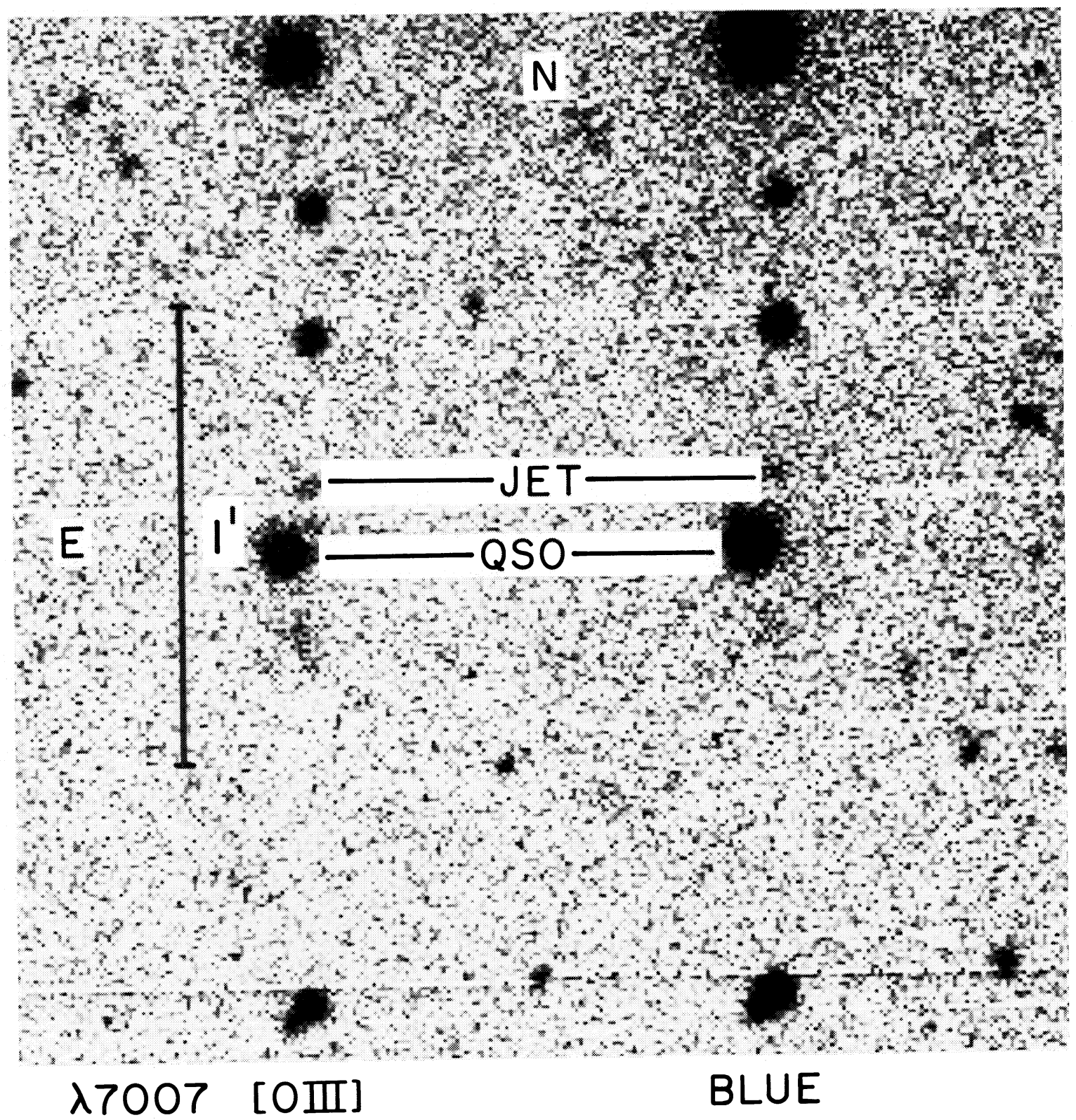

PKS $0812+02$

FIG. 11--Images in redshifted [O III] $\lambda 5007$ (left) and the blue (line-free) continuum (right) of the $z=0.4$ QSO PKS $0812+02$. Extended structure is apparent to the NW and $\mathrm{S}$, but in both cases a substantial fraction may be line emission.

KeEL (see 329, 543) 